\title{
Stability of Uncertain Impulsive Stochastic Genetic Regulatory Networks with Time-Varying Delay in the Leakage Term
}

\author{
Jun Li, ${ }^{1}$ Manfeng Hu, ${ }^{1,2}$ Jinde Cao, ${ }^{3,4}$ Yongqing Yang,, and Yinghua Jin ${ }^{1}$ \\ ${ }^{1}$ School of Science, Jiangnan University, Wuxi 214122, China \\ ${ }^{2}$ Key Laboratory of Advanced Process Control for Light Industry, Jiangnan University, Ministry of Education, Wuxi 214122, China \\ ${ }^{3}$ Department of Mathematics, Southeast University, Nanjing 210096, China \\ ${ }^{4}$ Department of Mathematics, Faculty of Science, King Abdulaziz University, Jeddah 21589, Saudi Arabia
}

Correspondence should be addressed to Manfeng Hu; humanfeng@jiangnan.edu.cn

Received 10 November 2013; Revised 19 January 2014; Accepted 22 January 2014; Published 6 April 2014

Academic Editor: Sanyi Tang

Copyright (C) 2014 Jun Li et al. This is an open access article distributed under the Creative Commons Attribution License, which permits unrestricted use, distribution, and reproduction in any medium, provided the original work is properly cited.

\begin{abstract}
This paper is concerned with the stability problem for a class of uncertain impulsive stochastic genetic regulatory networks (UISGRNs) with time-varying delays both in the leakage term and in the regulator function. By constructing a suitable LyapunovKrasovskii functional which uses the information on the lower bound of the delay sufficiently, a delay-dependent stability criterion is derived for the proposed UISGRNs model by using the free-weighting matrices method and convex combination technique. The conditions obtained here are expressed in terms of LMIs whose feasibility can be checked easily by MATLAB LMI control toolbox. In addition, three numerical examples are given to justify the obtained stability results.
\end{abstract}

\section{Introduction}

Genetic regulatory networks (GRNs) which govern many essential functions of living cells have received much attention due to their extensive applications in many practical systems, especially in the biology, engineering, and other research fields [1-6]. That is why GRNs have become a hot topic of research recently. Several computational models have been applied to investigate the behaviours of GRNs: Petri net models [7-9], Bayesian network models [10-12], the Boolean models [13-15], the differential equation models [16-18], and so forth. In this paper, we will use differential equation models to encode genetic regulatory networks. The rate of change in concentration of a particular transcript is given by an influence function of other RNA concentrations.

Time delay is an interesting feature of signal transmission and becomes one of the main sources for causing divergence, instability, and poor performances for networks stability. So, it is important to consider the delay effects on the dynamical behavior of GRNs. Up to now, in almost all existing works on modeling GRNs [5, 19-21], time delay is included in the regulator function to describe the existing time delays peculiar to transcription, translation, and translocation processes in genetic networks. Chen and Aihara [5] firstly proposed a delay differential equation model for GRNs and studied its stability problem. In [19], Ren and Cao studied the asymptotic and robust stability of GRNs with time-varying delays. In [20], Zhang et al. investigated the stability analysis for GRNs with random discrete delays and distributed delays. Hu et al. [21] proposed a GRNs model with hybrid regulatory mechanism and studied its stability problem. Recently, Gopalsamy [22] put forward a neural network model with the incorporation of time delays in the leakage terms (i.e., negative feedback or decay terms which widely appeared in the models of neural networks, population dynamics, and GRNs). Along this line, a time delay will be taken into consideration in the decay terms of our GRNs model and we also call it "leakage delay."

When modeling the GRNs, stochastic disturbance should be taken into consideration since molecular noise plays important roles in biological functions of GRNs in practice. In $[23,24]$, the authors studied the model of GRNs with stochastic disturbances. Moreover, impulsive effects are also likely to exist in the genetic networks systems [25]. In [26], Li and Sun researched the stability of GRNs under impulsive control. On the other hand, it is well known that the 
stability of well-designed GRNs may often be destroyed by its unavoidable uncertainty in practice. In $[27,28]$, the authors investigated the stability for uncertain GRNs with interval time-varying delays. In $[29,30]$, the authors researched the stability problem of GRNs with stochastic disturbance and parameter uncertainties, simultaneously. In [31], Sakthivel et al. dealt with the asymptotic stability of delayed GRNs with both stochastic disturbance and impulsive effects. However, so far there has been very little published concerning the stability problem for GRNs with leakage delay, impulsive effects, stochastic disturbances, and parameter uncertainties, simultaneously.

Motivated by the above discussion, the stability analysis for UISGRNs with time-varying delays in the leakage term requires further consideration. By constructing a suitable Lyapunov-Krasovskii functional which uses the information on the lower bound of all the delays, the derived conditions are expressed in terms of LMIs whose feasibility can be easily checked by using numerically efficient MATLAB LMI control toolbox. It is believed that the result is meaningful and useful for the design and applications of UISGRNs. Finally, numerical examples are provided to show the usefulness of the derived LMI-based stability conditions.

Notations. Throughout this paper, $\mathbb{R}^{n}$ and $\mathbb{R}^{n \times n}$ denote, respectively, the $n$-dimensional Euclidean space and the set of all $n \times n$ real matrices. The superscript $T$ denotes the transposition and the notation $X \geq Y$ (resp., $X>Y$ ), where $X$ and $Y$ are symmetric matrices, and it means that $X-Y$ is positive semidefinite (resp., positive definite). Diag(·) denotes the diagonal matrix, and $\operatorname{col}\{\cdot\}$ means a column vector. In symmetric block matrices, we use an asterisk $(*)$ to represent a term that is induced by symmetry. Matrices, if their dimensions are not explicitly stated, are assumed to be compatible for algebraic operations.

\section{Problem Formulation and Preliminaries}

In this paper, we consider the following model:

$$
\begin{aligned}
d m_{i}(t)=( & -a_{i} m_{i}\left(t-d_{1}(t)\right) \\
& +b_{i}\left(p_{1}(t-\sigma(t)), p_{2}(t-\sigma(t)), \ldots,\right. \\
& \left.\left.p_{n}(t-\sigma(t))\right)\right) d t \\
& +\eta_{i}\left(t, m_{i}\left(t-d_{1}(t)\right), p_{i}(t-\sigma(t))\right) d \omega(t), \\
\left.\Delta m_{i}(t)\right|_{t=t_{k}}= & m_{i}\left(t_{k}\right)-m_{i}\left(t_{k}^{-}\right)=J_{k}\left(m_{i}\left(t_{k}^{-}\right)\right), \\
& k t_{k}, \\
d p_{i}(t)= & \left(-c_{i} p_{i}\left(t-d_{2}(t)\right)+l_{i} m_{i}(t-\tau(t))\right) d t \\
& +\eta_{i}\left(t, m_{i}(t-\tau(t)), p_{i}\left(t-d_{2}(t)\right)\right) d \omega(t),
\end{aligned}
$$

$$
\begin{array}{r}
\left.\Delta p_{i}(t)\right|_{t=t_{k}}=p_{i}\left(t_{k}\right)-p_{i}\left(t_{k}^{-}\right)=J_{k}\left(p_{i}\left(t_{k}^{-}\right)\right), \\
k \in Z^{+}, \quad t=t_{k} \quad i=1,2, \ldots, n,
\end{array}
$$

where $m_{i}(t), p_{i}(t)$ are concentrations of mRNA and protein of the $i$ th node at time $t$, respectively, $a_{i}$ and $c_{i}$ are positive real numbers that are the degradation rates of the mRNA and protein, $l_{i}$ is a positive constant that represents the translation rate, and $b_{i}(\cdot)$ is the regulatory function of the $i$ th gene. The first term in the first and third equations of the right side of (1) is called decay term and $d_{i}(t), i=1,2$, is called "leakage delay" as discussed in the Introduction. The regulatory function is of the form $b_{i}\left(p_{1}(t), p_{2}(t), \ldots, p_{n}(t)\right)=\sum_{j=1}^{n} b_{i j}\left(p_{j}(t)\right)$, which is called SUM logic [32]. The stochastic disturbance $\omega(t)$ is one-dimensional Brownian motion defined on a complete probability space $(\Omega, \mathscr{F}, P)$ with a natural filtration $\mathscr{F}_{t \geq 0}$ and $\eta_{i}\left(t, m_{i}(t-\tau(t)), p_{i}\left(t-d_{2}(t)\right)\right) \in \mathbb{R}$ is the noise intensity.

The function $b_{i j}\left(p_{j}(t)\right)$ is a monotonic function of the Hill form as follows:

$$
\begin{aligned}
& b_{i j}\left(p_{j}(t)\right) \\
& =\left\{\begin{array}{l}
\alpha_{i j} \frac{\left(p_{j}(t) / \beta_{j}\right)^{H_{j}}}{1+\left(p_{j}(t) / \beta_{j}\right)^{H_{j}}} \\
\text { if transcription factor } j \text { is an activator } \\
\text { of gene } i, \\
\alpha_{i j} \frac{1}{1+\left(p_{j}(t) / \beta_{j}\right)^{H_{j}}} \\
\text { if transcription factor } j \text { is an repressor } \\
\text { of gene } i,
\end{array}\right.
\end{aligned}
$$

where $H_{j}$ is the Hill coefficient, $\beta_{j}$ is a positive constant, and $\alpha_{i j}$ is the dimensionless transcriptional rate of transcription factor $j$ to gene $i$, which is a bounded constant. Therefore, (1) can be rewritten into the following form:

$$
\begin{aligned}
d m_{i}(t)= & \left(-a_{i} m_{i}\left(t-d_{1}(t)\right)\right. \\
& \left.+\sum_{j=1}^{n} v_{i j} h_{j}\left(p_{j}(t-\sigma(t))\right)+u_{i}\right) d t \\
& +\eta_{i}\left(t, m_{i}\left(t-d_{1}(t)\right), p_{i}(t-\sigma(t))\right) d \omega(t), \\
&
\end{aligned}
$$

$$
\begin{aligned}
&\left.\Delta m_{i}(t)\right|_{t=t_{k}}= m_{i}\left(t_{k}\right)-m_{i}\left(t_{k}^{-}\right)=J_{k}\left(m_{i}\left(t_{k}^{-}\right)\right), \\
& k \in Z^{+}, \quad t=t_{k}, \\
& d p_{i}(t)=\left(-c_{i} p_{i}\left(t-d_{2}(t)\right)+l_{i} m_{i}(t-\tau(t))\right) d t \\
&+\eta_{i}\left(t, m_{i}(t-\tau(t)), p_{i}\left(t-d_{2}(t)\right)\right) d \omega(t),
\end{aligned}
$$




$$
\begin{array}{r}
\left.\Delta p_{i}(t)\right|_{t=t_{k}}=p_{i}\left(t_{k}\right)-p_{i}\left(t_{k}^{-}\right)=J_{k}\left(p_{i}\left(t_{k}^{-}\right)\right), \\
k \in Z^{+}, \quad t=t_{k}, \quad i=1,2, \ldots, n,
\end{array}
$$

where $h_{j}(x)=\left(x / \beta_{j}\right)^{H_{j}} /\left(1+\left(x / \beta_{j}\right)^{H_{j}}\right), u_{i}=\Sigma_{j \in I_{i}} \alpha_{i j}$ is defined as a basal rate, and $I_{i}$ is the set of all the $j$ which is a repressor of gene $i$. The matrix $W=\left(v_{i j}\right) \in \mathbb{R}^{n \times n}$ of the genetic network is defined as follows:

$v_{i j}=\left\{\begin{array}{l}\alpha_{i j}, \\ \text { if transcription factor } j \text { is an activator of gene } i, \\ 0, \\ \text { if there is no link from node } j \text { to node } i \\ -\alpha_{i j}, \\ \text { if transcription factor } j \text { is a repressor of gene } i\end{array}\right.$

Rewriting system (3) into compact matrix form, we obtain

$$
\begin{gathered}
d m(t)=\left(-A m\left(t-d_{1}(t)\right)+W h(p(t-\sigma(t)))+u\right) d t \\
+\eta\left(t, m\left(t-d_{1}(t)\right), p(t-\sigma(t))\right) d \omega(t), \\
t \neq t_{k}, \\
\left.\Delta m(t)\right|_{t=t_{k}}=m\left(t_{k}\right)-m\left(t_{k}^{-}\right)=J_{k}\left(m\left(t_{k}^{-}\right)\right), \\
k \in Z^{+}, \quad t=t_{k}, \\
\begin{aligned}
& d p(t)=\left(-C p\left(t-d_{2}(t)\right)+L m(t-\tau(t))\right) d t \\
&+\eta\left(t, m(t-\tau(t)), p\left(t-d_{2}(t)\right)\right) d \omega(t), \\
&\left.\Delta p(t)\right|_{t=t_{k}}= p\left(t_{k}\right)-p\left(t_{k}^{-}\right)=J_{k}\left(p\left(t_{k}^{-}\right)\right), \\
& k \in Z^{+}, \quad t=t_{k},
\end{aligned}
\end{gathered}
$$

where $A=\operatorname{diag}\left(a_{1}, a_{2}, \ldots, a_{n}\right), u=\operatorname{col}\left\{u_{1}, u_{2}, \ldots, u_{n}\right\}, C=$ $\operatorname{diag}\left(c_{1}, c_{2}, \ldots, c_{n}\right), L=\operatorname{diag}\left(l_{1}, l_{2}, \ldots, l_{n}\right), m(t)=\operatorname{col}\left\{m_{1}(t)\right.$, $\left.m_{2}(t), \ldots, m_{n}(t)\right\}, p(t)=\operatorname{col}\left\{p_{1}(t), p_{2}(t), \ldots, p_{n}(t)\right\}, h(p(t))$ $=\operatorname{col}\left\{h_{1}\left(p_{1}(t)\right), h_{2}\left(p_{2}(t)\right), \ldots, h_{n}\left(p_{n}(t)\right)\right\}$, and $\eta(t, x, y)=$ $\operatorname{col}\left\{\eta_{1}(t, x, y), \eta_{2}(t, x, y), \ldots, \eta_{n}(t, x, y)\right\}$.

Let $\left(m^{*}, p^{*}\right)$ be a nonnegative equilibrium point of the system (5). In the following, we will always shift the equilibrium point $\left(m^{*}, p^{*}\right)$ to the origin by letting $x(t)=$ $m(t)-m^{*}, y(t)=p(t)-p^{*}$. Hence, system (5) can be transformed into the following form:

$$
\begin{aligned}
d x(t)= & \left(-A x\left(t-d_{1}(t)\right)+W f(y(t-\sigma(t)))\right) d t \\
& +\eta\left(t, x\left(t-d_{1}(t)\right), y(t-\sigma(t))\right) d \omega(t),
\end{aligned}
$$$$
t \neq t_{k}
$$

$$
\begin{aligned}
&\left.\Delta x(t)\right|_{t=t_{k}}= x\left(t_{k}\right)-x\left(t_{k}^{-}\right)=J_{k}\left(x\left(t_{k}^{-}\right)\right), \\
& k \in Z^{+}, \quad t=t_{k}, \\
& d y(t)=\left(-C y\left(t-d_{2}(t)\right)+L x(t-\tau(t))\right) d t \\
&+\eta\left(t, x(t-\tau(t)), y\left(t-d_{2}(t)\right)\right) d \omega(t), \\
& t \neq t_{k},
\end{aligned}
$$

$$
\left.\Delta y(t)\right|_{t=t_{k}}=y\left(t_{k}\right)-y\left(t_{k}^{-}\right)=J_{k}\left(y\left(t_{k}^{-}\right)\right),
$$

$$
k \in Z^{+}, \quad t=t_{k}
$$

where $x\left(t-d_{1}(t)\right)=\operatorname{col}\left\{x_{1}\left(t-d_{1}(t)\right), x_{2}\left(t-d_{1}(t)\right), \ldots, x_{n}(t-\right.$ $\left.\left.d_{1}(t)\right)\right\} \in \mathbb{R}^{n}, y\left(t-d_{2}(t)\right)=\operatorname{col}\left\{y_{1}\left(t-d_{2}(t)\right), y_{2}\left(t-d_{2}(t)\right)\right.$, $\left.\ldots, y_{n}\left(t-d_{2}(t)\right)\right\} \in \mathbb{R}^{n}, f(y(t))=\operatorname{col}\left\{f_{1}\left(y_{1}(t)\right), f_{2}\left(y_{2}(t)\right)\right.$, $\left.\ldots, f_{n}\left(y_{n}(t)\right)\right\} \in \mathbb{R}^{n}$, the function $f_{j}\left(y_{j}(t)\right)=h_{j}\left(y_{j}(t)+p_{j}^{*}\right)-$ $h_{j}\left(p_{j}^{*}\right)$, and obviously $\mathbf{f}_{\mathbf{j}}(\mathbf{0})=\mathbf{0}$.

Due to the fact that $h_{j}$ is a monotonically increasing function with saturation, from the relationship of $f(\cdot)$ and $h(\cdot)$, we know that, for any $y_{i} \in R$,

$$
\gamma_{i} \leq \frac{f_{i}\left(y_{i}\right)}{y_{i}} \leq \alpha_{i}, \quad i=1,2, \ldots, n,
$$

where $\gamma_{i}$ and $\alpha_{i}$ are known constant scalars.

Taking parameter uncertainties into the GRNs model (6), we consider the following UISGRNs model:

$$
\begin{aligned}
& d x(t)=\left(-(A+\Delta A) x\left(t-d_{1}(t)\right)+(W+\Delta W)\right. \\
& \times f(y(t-\sigma(t)))) d t \\
& +\eta\left(t, x\left(t-d_{1}(t)\right), y(t-\sigma(t))\right) d \omega(t), \\
& t \neq t_{k}, \\
& \left.\Delta x(t)\right|_{t=t_{k}}=x\left(t_{k}\right)-x\left(t_{k}^{-}\right)=J_{k}\left(x\left(t_{k}^{-}\right)\right), \\
& k \in Z^{+}, \quad t=t_{k}, \\
& d y(t)=\left(-(C+\Delta C) y\left(t-d_{2}(t)\right)\right. \\
& +(L+\Delta L) x(t-\tau(t))) d t \\
& +\eta\left(t, x(t-\tau(t)), y\left(t-d_{2}(t)\right)\right) d \omega(t), \\
& t \neq t_{k}, \\
& \left.\Delta y(t)\right|_{t=t_{k}}=y\left(t_{k}\right)-y\left(t_{k}^{-}\right)=J_{k}\left(y\left(t_{k}^{-}\right)\right), \\
& k \in Z^{+}, \quad t=t_{k}, \\
& x_{0}=x(\theta)=\varphi(\theta), \quad y_{0}=y(\theta)=\psi(\theta), \quad \forall \theta \in[-\varepsilon, 0],
\end{aligned}
$$

where $\psi(\cdot)$ and $\varphi(\cdot)$ are the initial function which are continuously differentiable on $[-\varepsilon, 0]$ with $\varepsilon=\max \left\{h_{2}, h_{4}, h_{6}, h_{8}\right\}$. We extend $\varrho(\theta)$ on $\theta \in[-2 \varepsilon, 0]$ to satisfy $\|\varrho\|_{\varepsilon}=\|\varrho\|_{2 \varepsilon}$ with $\|\varrho\|_{\varepsilon}=\sup _{\theta \in[-\varepsilon, 0]}\|\varrho(\theta)\|,\|\varrho\|_{2 \varepsilon}=\sup _{\theta \in[-2 \varepsilon, 0]}\|\varrho(\theta)\|$, where $\varrho=\{\psi, \varphi\}$. 
Moreover, the noise intensity $\eta$ satisfies

$$
\begin{aligned}
\operatorname{tr}[ & \eta^{T}\left(t, x(t-\tau(t)), y\left(t-d_{2}(t)\right)\right) \\
& \left.\times \eta\left(t, x(t-\tau(t)), y\left(t-d_{2}(t)\right)\right)\right] \\
\leq & x^{T}(t-\tau(t)) \Sigma_{1}^{T} \Sigma_{1} x(t-\tau(t)) \\
& +y^{T}\left(t-d_{2}(t)\right) \Sigma_{2}^{T} \Sigma_{2} y_{2}(t-d(t)),
\end{aligned}
$$

where $\Sigma_{1}$ and $\Sigma_{2}$ are constant matrices with appropriate dimensions.

In order to obtain our main theorem, the following assumptions and lemmas for the system (8) are always made throughout this paper.

Assumption 1. The parametric uncertainties $\Delta A(t), \Delta W(t)$, $\Delta C(t)$, and $\Delta L(t)$ satisfy

$$
\begin{aligned}
\Delta A(t) & =G_{1} F_{1}(t) H_{a} \\
\Delta W(t) & =G_{1} F_{1}(t) H_{w} \\
\Delta C(t) & =G_{2} F_{2}(t) H_{c} \\
\Delta L(t) & =G_{2} F_{2}(t) H_{l},
\end{aligned}
$$

where $G_{1}, G_{2}, H_{a}, H_{w}, H_{c}$, and $H_{l}$ are some given constant matrices with appropriate dimensions and $F_{i}(t)$ satisfies $F_{i}^{T}(t) F_{i}(t) \leq I, i=1,2$, for any $t>0$.

Assumption 2. $d_{1}(t), d_{2}(t), \tau(t)$, and $\sigma(t)$ are the time-varying delays satisfying

$$
\begin{aligned}
0 \leq h_{1} \leq d_{1}(t) \leq h_{2}, & 0 \leq \dot{d}_{1}(t) \leq d_{1}<\infty, \\
0 \leq h_{3} \leq \sigma(t) \leq h_{4}, & 0 \leq \dot{\sigma}(t) \leq \sigma<\infty, \\
0 \leq h_{5} \leq d_{2}(t) \leq h_{6}, & 0 \leq \dot{d}_{2}(t) \leq d_{2}<\infty \\
0 \leq h_{7} \leq \tau(t) \leq h_{8}, & 0 \leq \dot{\tau}(t) \leq \tau<\infty, \\
h_{12}=h_{2}-h_{1}, & h_{34}=h_{4}-h_{3}, \\
h_{56}=h_{6}-h_{5}, & h_{78}=h_{8}-h_{7} .
\end{aligned}
$$

Lemma 3 (Schur complement, see [30]). For a given matrix

$$
\left(\begin{array}{cc}
Q(x) & S(x) \\
S^{T}(x) & R(x)
\end{array}\right)>0
$$

where

$$
Q(x)=Q^{T}(x), \quad R(x)=R^{T}(x),
$$

and a vector function $w(x):[0, r] \rightarrow R^{n}$ such that the intergrals concerned as well defined, then the following holds:

(i) $Q(x)>0, R(x)-S^{T}(x) Q(x)^{-1} S(x)>0$,

(ii) $R(x)>0, Q(x)-S(x) R(x)^{-1} S^{T}(x)>0$.
Lemma 4 (see [33]). For any constant symmetric matrix $M>$ $0, \operatorname{scalar} \gamma>0$,

$$
\left[\int_{0}^{\gamma} \omega(s) d s\right]^{T} M\left[\int_{0}^{\gamma} \omega(s) d s\right] \leq \gamma \int_{0}^{\gamma} \omega^{T}(s) M \omega(s) d s .
$$

Lemma 5 (see [29]). For any vectors $a, b \in \mathbb{R}^{n}$ and any positive matrix $Y$ satisfying:

$$
\pm 2 a^{T} b \leq a^{T} Y a+b^{T} Y^{-1} b
$$

\section{Main Result}

In this section, mean square stability result for model (8) is summarized in the following theorem.

Theorem 6. If (7), (9), and Assumptions 1 and 2 hold, there exist $\mu \geq 0, \lambda \geq 0, \rho_{1}>0, \rho_{2}>0, \chi_{\text {im }} \in[\mathbf{0}, \mathbf{1}], k=0,1, \ldots, r+$ 2 , and $i=1, \ldots, n, m \in Z^{+}$, such that the impulsive operator $J_{m}(\cdot)$ satisfies $J_{i m}\left(x_{i}\left(t_{m}\right)\right)=-\chi_{i m} x_{i}\left(t_{m}\right)$. The system (8) is stable in the mean square if there exist real matrices $P_{1}>0, P_{2}>0$, $Q_{i}>0(i=1,2, \ldots, 16), Z_{i}>0(i=1,2, \ldots, 8), V_{1}>0$, and $V_{2}>0$, diagonal matrices $Y_{i}>0(i=1,2, \ldots, 6)$, and any matrices $N_{11}, N_{12}, N_{21}, N_{22}, M_{11}, M_{12}, M_{21}, M_{22}, M_{31}, M_{32}$, $S_{11}, S_{12}, S_{21}, S_{22}, S_{31}, S_{32}, E_{11}, E_{12}, E_{21}, E_{22}, E_{31}$, and $E_{32}$ to satisfy the following ten linear matrix inequalities:

$$
\begin{aligned}
& P_{1}+V_{1}<\rho_{1} I, \\
& P_{2}+V_{2}<\rho_{2} I \text {, } \\
& \phi_{1}=\left[\begin{array}{cc}
\Xi & h_{12} N_{2} \\
* & -h_{12} Z_{2}
\end{array}\right]<0, \\
& \phi_{2}=\left[\begin{array}{cc}
\Xi & h_{12} N_{3} \\
* & -h_{12} Z_{2}
\end{array}\right]<0, \\
& \phi_{3}=\left[\begin{array}{cc}
\Xi & h_{34} M_{2} \\
* & -h_{34} Z_{4}
\end{array}\right]<0, \\
& \phi_{4}=\left[\begin{array}{cc}
\Xi & h_{34} M_{3} \\
* & -h_{34} Z_{4}
\end{array}\right]<0, \\
& \phi_{5}=\left[\begin{array}{cc}
\Xi & h_{56} S_{2} \\
* & -h_{56} Z_{6}
\end{array}\right]<0, \\
& \phi_{6}=\left[\begin{array}{cc}
\Xi & h_{56} S_{3} \\
* & -h_{56} Z_{6}
\end{array}\right]<0 \text {, } \\
& \phi_{7}=\left[\begin{array}{cc}
\Xi & h_{78} E_{2} \\
* & -h_{78} Z_{8}
\end{array}\right]<0, \\
& \phi_{8}=\left[\begin{array}{cc}
\Xi & h_{78} E_{3} \\
* & -h_{78} Z_{8}
\end{array}\right]<0,
\end{aligned}
$$


where

$$
\begin{aligned}
& \Xi=\frac{1}{4}\left[\begin{array}{ccccccc}
\phi & T_{1} X & T_{2} Y & h_{1} N_{1} & h_{3} M_{1} & h_{5} S_{1} & h_{7} E_{1} \\
* & -X & 0 & 0 & 0 & 0 & 0 \\
* & 0 & -Y & 0 & 0 & 0 & 0 \\
* & 0 & 0 & -h_{1} Z_{1} & 0 & 0 & 0 \\
* & 0 & 0 & 0 & -h_{3} Z_{3} & 0 & 0 \\
* & 0 & 0 & 0 & 0 & h_{5} Z_{5} & 0 \\
* & 0 & 0 & 0 & 0 & 0 & -h_{7} Z_{7}
\end{array}\right] \\
& \phi=\left[\begin{array}{ll}
\phi_{9} & \phi_{10} \\
* & \phi_{11}
\end{array}\right], \\
& \phi_{9}=\left[\begin{array}{cccccccccc}
\phi_{1,1} & \phi_{1,2} & N_{21} & -N_{31} & \phi_{1,5} & E_{21} & E_{31} & S_{11}+M_{11} & \phi_{1,9} & M_{21} \\
* & \phi_{2,2} & N_{22} & -N_{32} & \phi_{2,5} & E_{22} & -E_{32} & M_{12}+S_{12} & \phi_{2,9} & M_{22} \\
* & * & \phi_{3,3} & 0 & 0 & 0 & 0 & 0 & 0 & 0 \\
* & * & * & -Q_{4} & 0 & 0 & 0 & 0 & 0 & 0 \\
* & * & * & * & \phi_{5,5} & 0 & 0 & \phi_{5,8} & 0 & 0 \\
* & * & * & * & * & \phi_{6,6} & 0 & 0 & 0 & 0 \\
* & * & * & * & * & * & -Q_{16} & 0 & 0 & 0 \\
* & * & * & * & * & * & * & \phi_{8,8} & 0 & 0 \\
* & * & * & * & * & * & * & * & \phi_{9,9} & 0 \\
* & * & * & * & * & * & * & * & * & \phi_{10,10}
\end{array}\right], \\
& \phi_{10}=\left[\begin{array}{cccccccccc}
-M_{31} & \phi_{1,12} & S_{12} & -S_{31} & \phi_{1,15} & 0 & \phi_{1,17} & 0 & 0 & 0 \\
-M_{32} & \phi_{2,12} & S_{22} & -S_{32} & \phi_{2,15} & 0 & 0 & \phi_{2,18} & 0 & 0 \\
0 & 0 & 0 & 0 & 0 & 0 & 0 & 0 & 0 & 0 \\
0 & 0 & 0 & 0 & 0 & 0 & 0 & 0 & 0 & 0 \\
0 & 0 & 0 & 0 & 0 & \phi_{5,16} & 0 & 0 & \phi_{5,19} & 0 \\
0 & 0 & 0 & 0 & 0 & 0 & 0 & 0 & 0 & 0 \\
0 & 0 & 0 & 0 & 0 & 0 & 0 & 0 & 0 & 0 \\
0 & \phi_{8,12} & 0 & 0 & 0 & \phi_{8,16} & 0 & 0 & 0 & 0 \\
0 & 0 & 0 & 0 & 0 & 0 & \phi_{9,17} & 0 & 0 & 0 \\
0 & 0 & 0 & 0 & 0 & 0 & 0 & 0 & 0 & 0
\end{array}\right], \\
& \phi_{11}=\left[\begin{array}{cccccccccc}
-Q_{8} & 0 & 0 & 0 & 0 & 0 & 0 & 0 & 0 & 0 \\
* & \phi_{12,12} & 0 & 0 & 0 & \phi_{12,16} & 0 & 0 & 0 & \phi_{12,20} \\
* & * & \phi_{13,13} & 0 & 0 & 0 & 0 & 0 & 0 & 0 \\
* & * & * & -Q_{12} & 0 & 0 & 0 & 0 & 0 & 0 \\
* & * & * & * & \phi_{15,15} & 0 & \phi_{15,17} & 0 & 0 & 0 \\
* & * & * & * & * & \phi_{16,16} & 0 & 0 & 0 & 0 \\
* & * & * & * & * & * & \phi_{17,17} & 0 & 0 & 0 \\
* & * & * & * & * & * & * & \phi_{18,18} & 0 & 0 \\
* & * & * & * & * & * & * & * & \phi_{19,19} & 0 \\
* & * & * & * & * & * & * & * & * & \phi_{20,20}
\end{array}\right], \\
& J_{\text {im }}\left(x\left(t_{m}\right)\right)=\left(J_{1 m}\left(x_{1}\left(t_{m}\right)\right), \ldots, J_{n m}\left(x_{n}\left(t_{m}\right)\right)\right)^{T}, \quad \phi_{1,1}=Q_{3}+Q_{15}+2 N_{11}+2 E_{11}-T Y_{6} \Sigma, \\
& \phi_{1,2}=-P_{1} A-P_{1} G_{1} F_{1} H_{a}-T V_{2} A-T V_{2} G_{1} F_{1} H_{a}-N_{11}+N_{12}+N_{13}-N_{21}+E_{12}, \\
& \phi_{1,5}=-E_{11}+E_{31}-E_{21}, \quad \phi_{1,9}=-M_{11}+M_{31}-M_{21}, \quad \phi_{1,12}=-S_{11}+S_{31}-S_{21} \text {, } \\
& \phi_{1,15}=\frac{1}{2} Y_{6}(T+\Sigma), \quad \phi_{1,17}=-T V_{2} W-T V_{2} G_{1} F_{1} H_{w}+P_{1} W+P_{1} G_{1} F_{1} H_{w} \\
& \phi_{2,2}=-\left(1-d_{1}\right) Q_{1}-2 N_{12}-2 N_{22}+2 M_{32}+\rho_{1} \Sigma_{1}^{T} \Sigma_{1}-T Y_{2} \Sigma, \quad \phi_{2,5}=-E_{12}+E_{32}-E_{22} \text {, } \\
& \phi_{2,9}=-M_{12}+M_{32}-M_{22}, \quad \phi_{2,12}=-S_{12}+S_{32}-S_{22}, \quad \phi_{2,15}=-V_{2} A-V_{2} G_{1} F_{1} H_{a}, \\
& \phi_{2,18}=\frac{1}{2} Y_{2}(T+\Sigma), \quad \phi_{3,3}=Q_{1}+Q_{4}-Q_{3}, \quad \phi_{5,5}=-(1-\tau) Q_{13}+\rho_{2} \Sigma_{4} \Sigma_{4}-T Y_{5} \Sigma
\end{aligned}
$$




$$
\begin{aligned}
& \phi_{5,8}=-T V_{1} L-T V_{1} G_{2} F_{2} H_{l}+P_{2} L+P_{2} G_{2} F_{2} H_{l}, \quad \phi_{5,16}=V_{1} L+V_{1} G_{2} F_{2} H_{l}, \\
& \phi_{5,19}=\frac{1}{2} Y_{5}(T+\Sigma), \quad \phi_{6,6}=Q_{13}+Q_{16}-Q_{15}, \quad \phi_{8,8}=Q_{7}+Q_{11}-T Y_{1} \Sigma, \\
& \phi_{8,12}=-P_{2} C-P_{2} G_{2} F_{2} H_{c}+T V_{1} C+T V_{1} G_{2} F_{2} H_{c}, \quad \phi_{8,16}=\frac{1}{2} Y_{1}(T+\Sigma), \\
& \phi_{9,9}=-(1-\sigma) Q_{5}+\rho_{1} \Sigma_{2} \Sigma_{2}-T Y_{3} \Sigma, \quad \phi_{9,17}=\frac{1}{2} Y_{3}(T+\Sigma), \quad \phi_{10,10}=Q_{5}-Q_{7}+Q_{8}, \\
& \phi_{12,12}=-\left(1-d_{2}\right) Q_{9}+\rho_{2} \Sigma_{3} \Sigma_{3}-T Y_{4} \Sigma, \quad \phi_{12,16}=-V_{1} C-V_{1} G_{2} F_{2} H_{c}, \\
& \phi_{12,20}=\frac{1}{2} Y_{4}(T+\Sigma), \quad \phi_{13,13}=Q_{9}-Q_{11}+Q_{12}, \quad \phi_{15,15}=Q_{14}-Y_{6}+Q_{2}, \\
& \phi_{15,17}=V_{2} W+V_{2} G_{1} F_{1} H_{w}, \quad \phi_{16,16}=Q_{6}-Y_{1}+Q_{10}, \quad \phi_{17,17}=-(1-\sigma) Q_{6}-Y_{3}, \\
& \phi_{18,18}=-\left(1-d_{1}\right) Q_{2}-Y_{2}, \quad \phi_{19,19}=-(1-\tau) Q_{15}-Y_{5}, \quad \phi_{20,20}=-\left(1-d_{2}\right) Q_{10}-Y_{4}, \\
& X=h_{1} Z_{1}+h_{12} Z_{2}+h_{7} Z_{7}+h_{78} Z_{8}, \quad Y=h_{2} Z_{3}+h_{34} Z_{4}+h_{5} Z_{5}+h_{56} Z_{6} \text {, } \\
& T_{11}=\left[\begin{array}{llllllllll}
0 & -A-G_{1} F_{1} H_{a} & 0 & 0 & 0 & 0 & 0 & 0 & 0 & 0
\end{array}\right],
\end{aligned}
$$

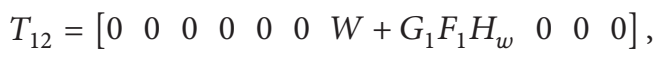

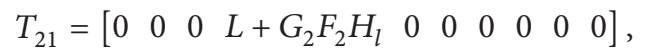

$$
\begin{aligned}
& T_{22}=\left[\begin{array}{lllllllllll}
0 & -C-G_{2} H_{2} H_{c} & 0 & 0 & 0 & 0 & 0 & 0 & 0 & 0
\end{array}\right] \text {, } \\
& T_{1}=\left[\begin{array}{ll}
T_{11} & T_{12}
\end{array}\right]^{T}, \quad T_{2}=\left[\begin{array}{ll}
T_{21} & T_{22}
\end{array}\right]^{T}, \\
& N_{1}=\left[\begin{array}{llllllllllllllllllll}
N_{11}^{T} & N_{12}^{T} & 0 & 0 & 0 & 0 & 0 & 0 & 0 & 0 & 0 & 0 & 0 & 0 & 0 & 0 & 0 & 0 & 0 & 0
\end{array}\right]^{T} \text {, } \\
& N_{2}=\left[\begin{array}{llllllllllllllllllll}
N_{21}^{T} & N_{22}^{T} & 0 & 0 & 0 & 0 & 0 & 0 & 0 & 0 & 0 & 0 & 0 & 0 & 0 & 0 & 0 & 0 & 0 & 0
\end{array}\right]^{T}, \\
& N_{3}=\left[\begin{array}{lllllllllllllllllllll}
N_{31}^{T} & N_{32}^{T} & 0 & 0 & 0 & 0 & 0 & 0 & 0 & 0 & 0 & 0 & 0 & 0 & 0 & 0 & 0 & 0 & 0 & 0
\end{array}\right]^{T}, \\
& S_{1}=\left[\begin{array}{llllllllllllllllllll}
S_{11}^{T} & S_{12}^{T} & 0 & 0 & 0 & 0 & 0 & 0 & 0 & 0 & 0 & 0 & 0 & 0 & 0 & 0 & 0 & 0 & 0 & 0
\end{array}\right]^{T} \text {, } \\
& S_{2}=\left[\begin{array}{llllllllllllllllllll}
S_{21}^{T} & S_{22}^{T} & 0 & 0 & 0 & 0 & 0 & 0 & 0 & 0 & 0 & 0 & 0 & 0 & 0 & 0 & 0 & 0 & 0 & 0
\end{array}\right]^{T} \text {, } \\
& S_{3}=\left[\begin{array}{llllllllllllllllllll}
S_{31}^{T} & S_{32}^{T} & 0 & 0 & 0 & 0 & 0 & 0 & 0 & 0 & 0 & 0 & 0 & 0 & 0 & 0 & 0 & 0 & 0 & 0
\end{array}\right]^{T} \text {, } \\
& M_{1}=\left[\begin{array}{llllllllllllllllllll}
M_{11}^{T} & M_{12}^{T} & 0 & 0 & 0 & 0 & 0 & 0 & 0 & 0 & 0 & 0 & 0 & 0 & 0 & 0 & 0 & 0 & 0 & 0
\end{array}\right]^{T} \text {, } \\
& M_{2}=\left[\begin{array}{llllllllllllllllllll}
M_{21}^{T} & M_{22}^{T} & 0 & 0 & 0 & 0 & 0 & 0 & 0 & 0 & 0 & 0 & 0 & 0 & 0 & 0 & 0 & 0 & 0 & 0
\end{array}\right]^{T} \text {, } \\
& M_{3}=\left[\begin{array}{llllllllllllllllllll}
M_{31}^{T} & M_{32}^{T} & 0 & 0 & 0 & 0 & 0 & 0 & 0 & 0 & 0 & 0 & 0 & 0 & 0 & 0 & 0 & 0 & 0 & 0
\end{array}\right]^{T}, \\
& E_{1}=\left[\begin{array}{llllllllllllllllllll}
E_{11}^{T} & E_{12}^{T} & 0 & 0 & 0 & 0 & 0 & 0 & 0 & 0 & 0 & 0 & 0 & 0 & 0 & 0 & 0 & 0 & 0 & 0
\end{array}\right]^{T}, \\
& E_{2}=\left[\begin{array}{llllllllllllllllllll}
E_{21}^{T} & E_{22}^{T} & 0 & 0 & 0 & 0 & 0 & 0 & 0 & 0 & 0 & 0 & 0 & 0 & 0 & 0 & 0 & 0 & 0 & 0
\end{array}\right]^{T} \text {, } \\
& E_{3}=\left[\begin{array}{llllllllllllllllllll}
E_{31}^{T} & E_{32}^{T} & 0 & 0 & 0 & 0 & 0 & 0 & 0 & 0 & 0 & 0 & 0 & 0 & 0 & 0 & 0 & 0 & 0 & 0
\end{array}\right]^{T} \text {, } \\
& T=\operatorname{diag}\left(\gamma_{1}, \gamma_{2}, \ldots, \gamma_{n}\right), \quad \Sigma=\operatorname{diag}\left(\alpha_{1}, \alpha_{2}, \ldots, \alpha_{n}\right) .
\end{aligned}
$$


Proof. We consider the following Lyapunov functional candidate for system (8):

$$
V\left(t, x_{t}, y_{t}\right)=V_{1}\left(t, x_{t}, y_{t}\right)+V_{2}\left(t, x_{t}, y_{t}\right)+V_{3}\left(t, x_{t}, y_{t}\right)
$$

where

$$
\begin{aligned}
& V_{1}\left(t, x_{t}, y_{t}\right)=2 \sum_{i=1}^{n} V_{1 i} \int_{0}^{y_{i}}\left(f_{i}(s)-\gamma_{i} s\right) d s \\
& +2 \sum_{i=1}^{n} V_{2 i} \int_{0}^{x_{i}}\left(f_{i}(s)-\gamma_{i} s\right) d s, \\
& V_{2}\left(t, x_{t}, y_{t}\right)=x^{T}(t) P_{1} x(t)+y^{T}(t) P_{2} y(t) \\
& +\int_{t-d_{1}(t)}^{t-h_{1}} x^{T}(s) Q_{1} x(s) d s \\
& +\int_{t-d_{1}(t)}^{t} f^{T}(x(s)) Q_{2} f(x(s)) d s \\
& +\int_{t-h_{1}}^{t} x^{T}(s) Q_{3} x(s) d s \\
& +\int_{t-h_{2}}^{t-h_{1}} x^{T}(s) Q_{4} x(s) d s \\
& +\int_{t-\sigma(t)}^{t-h_{3}} y^{T}(s) Q_{5} y(s) d s \\
& +\int_{t-\sigma(t)}^{t} f^{T}(y(s)) Q_{6} f(y(s)) d s \\
& +\int_{t-h_{3}}^{t} y^{T}(s) Q_{7} y(s) d s \\
& +\int_{t-h_{4}}^{t-h_{3}} y^{T}(s) Q_{8} y(s) d s \\
& +\int_{t-d_{2}(t)}^{t-h_{5}} y^{T}(s) Q_{9} y(s) d s \\
& +\int_{t-d_{2}(t)}^{t} f^{T}(y(s)) Q_{10} f(y(s)) d s \\
& +\int_{t-h_{5}}^{t} y^{T}(s) Q_{11} y(s) d s \\
& +\int_{t-h_{6}}^{t-h_{5}} y^{T}(s) Q_{12} y(s) d s \\
& +\int_{t-\tau(t)}^{t-h_{7}} x^{T}(s) Q_{13} x(s) d s \\
& +\int_{t-\tau(t)}^{t} f^{T}(x(s)) Q_{14} f(x(s)) d s
\end{aligned}
$$

$$
\begin{aligned}
V_{3}\left(t, x_{t}, y_{t}\right)= & \int_{-h_{1}}^{0} \int_{t+\theta}^{t} \dot{x}^{T}(s) Z_{1} \dot{x}(s) d s d \theta \\
& +\int_{-h_{2}}^{-h_{1}} \int_{t+\theta}^{t} \dot{x}^{T}(s) Z_{2} \dot{x}(s) d s d \theta \\
& +\int_{-h_{3}}^{0} \int_{t+\theta}^{t} \dot{y}^{T}(s) Z_{3} \dot{y}(s) d s d \theta \\
& +\int_{-h_{4}}^{-h_{3}} \int_{t+\theta}^{t} \dot{y}^{T}(s) Z_{4} \dot{y}(s) d s d \theta \\
& +\int_{-h_{5}}^{0} \int_{t+\theta}^{t} \dot{y}^{T}(s) Z_{5} \dot{y}(s) d s d \theta \\
& +\int_{-h_{6}}^{-h_{5}} \int_{t+\theta}^{t} \dot{y}^{T}(s) Z_{6} \dot{y}(s) d s d \theta \\
& +\int_{-h_{7}}^{0} \int_{t+\theta}^{t} \dot{x}^{T}(s) Z_{7} \dot{x}(s) d s d \theta \\
& +\int_{-h_{8}}^{-h_{7}} \int_{t+\theta}^{t} \dot{x}^{T}(s) Z_{8} \dot{x}(s) d s d \theta .
\end{aligned}
$$

Then, by Itô's differential formula, taking the derivative of $V(t)$ along the trajectories of the system (8), we can obtain the following stochastic differential [29]:

$$
\begin{aligned}
d V(t)= & \mathscr{F} V(t) d t \\
& +2 x^{T}(t) P_{1} \eta\left(t, x\left(t-d_{1}(t)\right), y(t-\sigma(t))\right) \\
& +2 y^{T} P_{2} \eta\left(t, x(t-\tau(t)), y\left(t-d_{2}(t)\right)\right),
\end{aligned}
$$

where $\mathscr{F}$ is the diffusion operator and

$$
\begin{aligned}
\mathscr{F} V\left(t, x_{t}, y_{t}\right)= & \mathscr{F} V_{1}\left(t, x_{t}, y_{t}\right) \\
& +\mathscr{F} V_{2}\left(t, x_{t}, y_{t}\right)+\mathscr{F} V_{3}\left(t, x_{t}, y_{t}\right),
\end{aligned}
$$

with

$$
\begin{gathered}
\mathscr{F} V_{1}\left(t, x_{t}, y_{t}\right)=2\left[f^{T}(y(t))-y^{T}(t) \Gamma\right] \\
\times V_{1}\left[-C y\left(t-d_{2}(t)\right)+L x(t-\tau(t))\right. \\
-G_{2} F_{2} H_{c} y\left(t-d_{2}(t)\right) \\
\left.+G_{2} F_{2} H_{l} x(t-\tau(t))\right]
\end{gathered}
$$




$$
\begin{aligned}
& +\operatorname{tr}\left[\eta^{T}\left(t, x(t-\tau(t)), y\left(t-d_{2}(t)\right)\right)\right. \\
& \left.\times V_{1} \eta\left(t, x(t-\tau(t)), y\left(t-d_{2}(t)\right)\right)\right] \\
& +2\left[f^{T}(x(t))-x^{T}(t) \Gamma\right] \\
& \times V_{2}\left[-A x\left(t-d_{1}(t)\right)+W f(y(t-\sigma(t)))\right. \\
& -G_{1} F_{1} H_{a} x\left(t-d_{1}(t)\right) \\
& \left.+G_{1} F_{1} H_{w} f(y(t-\sigma(t)))\right] \\
& +\operatorname{tr}\left[\eta^{T}\left(t, y(t-\sigma(t)), y\left(t-d_{1}(t)\right)\right)\right. \\
& \left.\times V_{2} \eta\left(t, y(t-\sigma(t)), y\left(t-d_{1}(t)\right)\right)\right], \\
& \mathscr{F} V_{2}\left(t, x_{t}, y_{t}\right) \\
& \leq 2 x^{T}(t) P_{1}\left[-A x\left(t-d_{1}(t)\right)\right. \\
& +W f(y(t-\sigma(t))) \\
& -G_{1} F_{1} H_{a} x\left(t-d_{1}(t)\right) \\
& \left.+G_{1} F_{1} H_{w} f(y(t-\sigma(t)))\right] \\
& +\operatorname{tr}\left[\eta^{T}\left(t, y(t-\sigma(t)), y\left(t-d_{1}(t)\right)\right)\right. \\
& \left.\times P_{1} \eta\left(t, y(t-\sigma(t)), y\left(t-d_{1}(t)\right)\right)\right] \\
& +2 y^{T}(t) P_{2}\left[-C y\left(t-d_{2}(t)\right)\right. \\
& +\operatorname{Lx}(t-\tau(t)) \\
& -G_{2} F_{2} H_{c} y\left(t-d_{2}(t)\right) \\
& \left.+G_{2} F_{2} H_{l} x(t-\tau(t))\right] \\
& +\operatorname{tr}\left[\eta^{T}\left(t, y(t-\tau(t)), y\left(t-d_{2}(t)\right)\right)\right. \\
& \left.\times P_{2} \eta\left(t, y(t-\tau(t)), y\left(t-d_{2}(t)\right)\right)\right] \\
& +x^{T}\left(t-h_{1}\right) Q_{1} x\left(t-h_{1}\right) \\
& -\left(1-d_{1}\right) x^{T}\left(t-d_{1}(t)\right) Q_{1} x\left(t-d_{1}(t)\right) \\
& +f^{T}(x(s)) Q_{2} f(x(s)) \\
& -\left(1-d_{1}\right) f^{T}\left(y\left(t-d_{1}(t)\right)\right) \\
& \times Q_{2} f\left(y\left(t-d_{1}(t)\right)\right) \\
& +x^{T}(t) Q_{3} x(t)-x^{T}\left(t-h_{1}\right) Q_{3} x\left(t-h_{1}\right) \\
& +x^{T}\left(t-h_{1}\right) Q_{4} x\left(t-h_{1}\right) \\
& -x^{T}\left(t-h_{2}\right) Q_{4} x\left(t-h_{2}\right) \\
& +y^{T}\left(t-h_{5}\right) Q_{5} x\left(t-h_{5}\right) \\
& -(1-\sigma) y^{T}(t-\sigma(t)) Q_{5} y(t-\sigma(t))
\end{aligned}
$$

$$
\begin{aligned}
& +f^{T}(y(t)) Q_{6} f(y(t)) \\
& -(1-\sigma) f\left(y^{T}(t-\sigma(t))\right) \\
& \times Q_{5} f(y(t-\sigma(t))) \\
& -y^{T}\left(t-h_{3}\right) Q_{7} y\left(t-h_{3}\right) \\
& +y^{T}(t) Q_{7} y(t)+y^{T}\left(t-h_{3}\right) Q_{8} y\left(t-h_{3}\right) \\
& -y^{T}\left(t-h_{4}\right) Q_{8} y\left(t-h_{4}\right) \\
& +y^{T}\left(t-h_{5}\right) Q_{9} y\left(t-h_{5}\right) \\
& -\left(1-d_{2}\right) y^{T}\left(t-d_{2}(t)\right) Q_{9} y\left(t-d_{2}(t)\right) \\
& +f^{T}(y(t)) Q_{10} f(y(t)) \\
& -\left(1-d_{2}\right) f^{T}\left(y\left(t-d_{2}(t)\right)\right) \\
& \times Q_{10} f\left(y\left(t-d_{2}(t)\right)\right) \\
& +y^{T} Q_{11} y(t)-y^{T}\left(t-h_{5}\right) Q_{11} y\left(t-h_{5}\right) \\
& +y^{T}\left(t-h_{5}\right) Q_{12} y\left(t-h_{5}\right) \\
& -y^{T}\left(t-h_{6}\right) Q_{12} y\left(t-h_{6}\right) \\
& +x^{T}\left(t-h_{7}\right) Q_{13} x\left(t-h_{7}\right) \\
& -(1-\tau) x^{T}(t-\tau(t)) Q_{13} x(t-\tau(t)) \\
& +f^{T}(x(t)) Q_{14} f(x(t)) \\
& -(1-\tau) f^{T}(x(t-\tau(t))) \\
& \times Q_{14} f(x(t-\tau(t)))+x^{T}(t) Q_{15} x(t) \\
& -x^{T}\left(t-h_{7}\right) Q_{15} x\left(t-h_{7}\right) \\
& +x^{T}\left(t-h_{7}\right) Q_{16} x\left(t-h_{7}\right) \\
& -x^{T}\left(t-h_{8}\right) Q_{16} x\left(t-h_{8}\right) \text {, } \\
& \mathscr{F} V_{3}\left(t, x_{t}, y_{t}\right) \\
& =h_{1} \dot{x}^{T}(t) Z_{1} \dot{x}(t) \\
& -\int_{t-h_{1}}^{t} \dot{x}^{T}(s) Z_{1} \dot{x}(s) d s \\
& +h_{12} \dot{x}^{T}(t) Z_{2} \dot{x}(t) \\
& -\int_{t-h_{2}}^{t-h_{1}} \dot{x}^{T}(s) Z_{2} \dot{x}(s) d s \\
& +h_{2} \dot{y}^{T}(t) Z_{3} \dot{y}(t) \\
& -\int_{t-h_{3}}^{t} \dot{y}^{T}(s) Z_{3} \dot{y}(s) d s
\end{aligned}
$$




$$
\begin{aligned}
& +h_{34} \dot{y}^{T}(t) Z_{4} \dot{y}(t) \\
& -\int_{t-h_{4}}^{t-h_{3}} \dot{y}^{T}(s) Z_{4} \dot{y}(s) d s \\
& +h_{5} \dot{y}^{T}(t) Z_{5} \dot{y}(t) \\
& -\int_{t-h_{5}}^{t} \dot{y}^{T}(s) Z_{5} \dot{y}(s) d s \\
& +h_{56} \dot{y}^{T}(t) Z_{6} \dot{y}(t) \\
& -\int_{t-h_{6}}^{t-h_{5}} \dot{y}^{T}(s) Z_{6} \dot{y}(s) d s \\
& +h_{7} \dot{x}^{T}(t) Z_{7} \dot{y}(t) \\
& -\int_{t-h_{7}}^{t} \dot{y}^{T}(s) Z_{7} \dot{y}(s) d s \\
& +h_{78} \dot{y}^{T}(s) Z_{8} \dot{y}(s) \\
& -\int_{t-h_{8}}^{t-h_{7}} \dot{y}^{T}(t) Z_{8} \dot{y}(t) d s .
\end{aligned}
$$

By Newton-Leibnitz formula, we have that

$$
2 \varepsilon^{T}(t) N_{1}\left[x(t)-x\left(t-h_{1}\right)-\int_{t-h_{1}}^{t} \dot{x}(s) d s\right]=0,
$$

$2 \varepsilon^{T}(t) N_{2}\left[x\left(t-h_{1}\right)-x\left(t-d_{1}(t)\right)-\int_{t-d_{1}(t)}^{t-h_{1}} \dot{x}(s) d s\right]=0$, $2 \varepsilon^{T}(t) N_{3}\left[x\left(t-d_{1}(t)\right)-x\left(t-h_{2}\right)-\int_{t-h_{2}}^{t-d_{1}(t)} \dot{x}(s) d s\right]=0$,

$$
2 \varepsilon^{T}(t) M_{1}\left[y(t)-y(t-\sigma(t))-\int_{t-\sigma(t)}^{t} \dot{y}(s) d s\right]=0,
$$

$2 \varepsilon^{T}(t) M_{2}\left[y\left(t-h_{3}\right)-y(t-\sigma(t))-\int_{t-\sigma(t)}^{t-h_{3}} \dot{y}(s) d s\right]=0$,

$2 \varepsilon^{T}(t) M_{3}\left[y(t-\sigma(t))-y\left(t-h_{4}\right)-\int_{t-h_{4}}^{t-\sigma(t)} \dot{y}(s) d s\right]=0$,

$$
2 \varepsilon^{T}(t) S_{1}\left[y(t)-y\left(t-d_{2}(t)\right)-\int_{t-d_{2}(t)}^{t} \dot{y}(s) d s\right]=0
$$

$2 \varepsilon^{T}(t) S_{2}\left[y\left(t-h_{5}\right)-y\left(t-d_{2}(t)\right)-\int_{t-d_{2}(t)}^{t-h_{5}} \dot{y}(s) d s\right]=0$,

$2 \varepsilon^{T}(t) S_{3}\left[y\left(t-d_{2}(t)\right)-y\left(t-h_{6}\right)-\int_{t-h_{6}}^{t-d_{2}(t)} \dot{y}(s) d s\right]=0$,

$$
2 \varepsilon^{T}(t) E_{1}\left[x(t)-x(t-\tau(t))-\int_{t-\tau(t)}^{t} \dot{x}(s) d s\right]=0
$$

$$
\begin{gathered}
2 \varepsilon^{T}(t) E_{2}\left[x\left(t-h_{7}\right)-x(t-\tau(t))-\int_{t-\tau(t)}^{t-h_{7}} \dot{x}(s) d s\right]=0 \\
2 \varepsilon^{T}(t) E_{3}\left[x\left(t-h_{8}\right)-x\left(t-h_{7}\right)-\int_{t-h_{8}}^{t-h_{7}} \dot{x}(s) d s\right]=0
\end{gathered}
$$

where

$$
\begin{gathered}
\varepsilon(t)=\left[\varepsilon_{1}(t), \varepsilon_{2}(t), \varepsilon_{3}(t), \varepsilon_{4}(t)\right]^{T}, \\
\varepsilon_{1}(t)=\left[x^{T}(t), x^{T}\left(t-d_{1}(t)\right), x^{T}\left(t-h_{1}\right),\right. \\
\left.x^{T}\left(t-h_{2}\right), x^{T}(t-\tau(t))\right], \\
\varepsilon_{2}(t)=\left[x^{T}\left(t-h_{7}\right), x^{T}\left(t-h_{8}\right), y^{T}(t),\right. \\
\varepsilon_{3}(t)=\left[y^{T}\left(t-h_{4}\right), y^{T}\left(t-d_{2}(t)\right), y^{T}\left(t-h_{5}\right),\right. \\
\left.y^{T}\left(t-h_{6}\right), f^{T}(x)\right], \\
\varepsilon_{4}(t)=\left[f^{T}(y), f^{T}(y-\sigma(t)), f^{T}\left(x-d_{1}(t)\right),\right. \\
\left.f^{T}(x-\tau(t)), f^{T}\left(y-d_{2}(t)\right)\right] .
\end{gathered}
$$

By using Lemmas 4 and 5, we have

$$
\begin{aligned}
&- 2 \varepsilon^{T}(t) N_{1} \int_{t-h_{1}}^{t} \dot{x}(s) d s \\
& \leq h_{1} \varepsilon^{T}(t) N_{1} Z_{1}^{-1} N_{1}^{T} \varepsilon(t) \\
&+\int_{t-h_{1}}^{t} \dot{x}^{T}(s) Z_{1} \dot{x}(s) d s, \\
&-2 \varepsilon^{T}(t) N_{2} \int_{t-d_{1}(t)}^{t-h_{1}} \dot{x}(s) d s \\
& \leq\left(d_{1}(t)-h_{1}\right) \varepsilon^{T}(t) N_{2} Z_{2}^{-1} N_{2}^{T} \varepsilon(t) \\
&+\int_{t-d_{1}(t)}^{t-h_{1}} \dot{x}^{T}(s) Z_{2} \dot{x}(s) d s \\
&-2 \varepsilon^{T}(t) N_{3} \int_{t-h_{2}}^{t-d_{1}(t)} \dot{x}(s) d s \\
& \leq\left(h_{2}-d_{1}(t)\right) \varepsilon^{T}(t) N_{3} Z_{2}^{-1} N_{3}^{T} \varepsilon(t) \\
&+\int_{t-h_{2}}^{t-d_{1}(t)} \dot{x}^{T}(s) Z_{2} \dot{x}(s) d s \\
&-2 \varepsilon^{T}(t) M_{1} \int_{t-h_{3}}^{t} \dot{y}(s) d s
\end{aligned}
$$




$$
\begin{aligned}
& \leq h_{3} \varepsilon^{T}(t) M_{1} Z_{3}^{-1} M_{1}^{T} \varepsilon(t) \\
& +\int_{t-h_{3}}^{t} \dot{y}^{T}(s) Z_{3} \dot{y}(s) d s \\
& -2 \varepsilon^{T}(t) M_{2} \int_{t-\sigma(t)}^{t-h_{3}} \dot{y}(s) d s \\
& \leq\left(\sigma(t)-h_{3}\right) \varepsilon^{T}(t) M_{2} Z_{4}^{-1} M_{2}^{T} \varepsilon(t) \\
& +\int_{t-\sigma(t)}^{t-h_{3}} \dot{y}^{T}(s) Z_{4} \dot{y}(s) d s, \\
& -2 \varepsilon^{T}(t) M_{3} \int_{t-h_{4}}^{t-\sigma(t)} \dot{y}(s) d s \\
& \leq\left(h_{4}-\sigma(t)\right) \varepsilon^{T}(t) M_{3} Z_{4}^{-1} M_{3}^{T} \varepsilon(t) \\
& +\int_{t-h_{4}}^{t-\sigma(t)} \dot{y}^{T}(s) Z_{4} \dot{y}(s) d s \\
& -2 \varepsilon^{T}(t) S_{1} \int_{t-h_{5}}^{t} \dot{y}(s) d s \\
& \leq h_{5} \varepsilon^{T}(t) S_{1} Z_{5}^{-1} S_{1}^{T} \varepsilon(t) \\
& +\int_{t-h_{5}}^{t} \dot{y}^{T}(s) Z_{5} \dot{y}(s) d s, \\
& -2 \varepsilon^{T}(t) S_{2} \int_{t-d_{2}(t)}^{t-h_{5}} \dot{y}(s) d s \\
& \leq\left(d_{2}(t)-h_{5}\right) \varepsilon^{T}(t) S_{2} Z_{6}^{-1} S_{2}^{T} \varepsilon(t) \\
& +\int_{t-d_{2}(t)}^{t-h_{5}} \dot{y}^{T}(s) Z_{6} \dot{y}(s) d s, \\
& -2 \varepsilon^{T}(t) S_{3} \int_{t-h_{6}}^{t-d_{2}(t)} \dot{y}(s) d s \\
& \leq\left(h_{6}-d_{2}(t)\right) \varepsilon^{T}(t) S_{3} Z_{6}^{-1} S_{3}^{T} \varepsilon(t) \\
& +\int_{t-h_{6}}^{t-d_{2}(t)} \dot{y}^{T}(s) Z_{6} \dot{y}(s) d s, \\
& -2 \varepsilon^{T}(t) E_{1} \int_{t-h_{7}}^{t} \dot{x}(s) d s \\
& \leq h_{7} \varepsilon^{T}(t) E_{1} Z_{7}^{-1} E_{1}^{T} \varepsilon(t) \\
& +\int_{t-h_{7}}^{t} \dot{x}^{T}(s) Z_{7} \dot{x}(s) d s, \\
& -2 \varepsilon^{T}(t) E_{2} \int_{t-\tau(t)}^{t-h_{7}} \dot{x}(s) d s \\
& \leq\left(\tau(t)-h_{7}\right) \varepsilon^{T}(t) E_{2} Z_{8}^{-1} E_{2}^{T} \varepsilon(t) \\
& +\int_{t-\tau(t)}^{t-h_{7}} \dot{x}^{T}(s) Z_{8} \dot{x}(s) d s,
\end{aligned}
$$

$$
\begin{aligned}
-2 \varepsilon^{T}(t) E_{3} \int_{t-h_{8}}^{t-\tau(t)} \dot{x}(s) d s \\
\leq\left(h_{8}-\tau(t)\right) \varepsilon^{T}(t) E_{3} Z_{8}^{-1} E_{3}^{T} \varepsilon(t) \\
\quad+\int_{t-h_{8}}^{t-\tau(t)} \dot{x}^{T}(s) Z_{8} \dot{x}(s) d s .
\end{aligned}
$$

It follows from (7) that

$$
\begin{aligned}
& 0=f^{T}(y(t)) Y_{1} f(y(t))-f^{T}(y(t)) Y_{1} f(y(t)) \\
& \leq-y^{T}(t) T Y_{1} \Sigma y(t)+y^{T}(t) Y_{1}(T+\Sigma) f(y(t)) \\
& -f^{T}(y(t)) Y_{1} f(y(t)) \\
& 0=f^{T}\left(y\left(t-d_{1}(t)\right)\right) Y_{2} f\left(y\left(t-d_{1}(t)\right)\right) \\
& -f^{T}\left(y\left(t-d_{1}(t)\right)\right) Y_{2} f\left(y\left(t-d_{1}(t)\right)\right) \\
& \leq-y^{T}\left(t-d_{1}(t)\right) T Y_{2} \Sigma y\left(t-d_{1}(t)\right) \\
& +y^{T}\left(t-d_{1}(t)\right) Y_{2}(T+\Sigma) f\left(y\left(t-d_{1}(t)\right)\right) \\
& -f^{T}\left(y\left(t-d_{1}(t)\right)\right) Y_{2} f\left(y\left(t-d_{1}(t)\right)\right) \text {, } \\
& 0=f^{T}(y(t-\sigma(t))) Y_{3} f(y(t-\sigma(t))) \\
& -f^{T}(y(t-\sigma(t))) Y_{3} f(y(t-\sigma(t))) \\
& \leq-y^{T}(t-\sigma(t)) T Y_{3} \Sigma y(t-\sigma(t)) \\
& +y^{T}(t-\sigma(t)) Y_{3}(T+\Sigma) f(y(t-\sigma(t))) \\
& -f^{T}(y(t-\sigma(t))) Y_{3} f(y(t-\sigma(t))), \\
& 0=f^{T}\left(y\left(t-d_{2}(t)\right)\right) Y_{4} f\left(y\left(t-d_{2}(t)\right)\right) \\
& -f^{T}\left(y\left(t-d_{2}(t)\right)\right) Y_{4} f\left(y\left(t-d_{2}(t)\right)\right) \\
& \leq-y^{T}\left(t-d_{2}(t)\right) T Y_{2} \Sigma y\left(t-d_{2}(t)\right) \\
& +y^{T}\left(t-d_{2}(t)\right) Y_{2}(T+\Sigma) f\left(y\left(t-d_{2}(t)\right)\right) \\
& -f^{T}\left(y\left(t-d_{2}(t)\right)\right) Y_{2} f\left(y\left(t-d_{2}(t)\right)\right), \\
& 0=f^{T}(y(t-\tau(t))) Y_{5} f(y(t-\tau(t))) \\
& -f^{T}(y(t-\tau(t))) Y_{5} f(y(t-\tau(t))) \\
& \leq-y^{T}(t-\tau(t)) T Y_{5} \Sigma y(t-\tau(t)) \\
& +y^{T}(t-\tau(t)) Y_{5}(T+\Sigma) f(y(t-\tau(t))) \\
& -f^{T}(y(t-\tau(t))) Y_{5} f(y(t-\tau(t))), \\
& 0=f^{T}(x(t)) Y_{6} f(x(t))-f^{T}(x(t)) Y_{6} f(x(t)) \\
& \leq-x^{T}(t) T Y_{6} \Sigma x(t)+x^{T}(t) Y_{6}(T+\Sigma) f(x(t)) \\
& -f^{T}(x(t)) Y_{6} f(x(t)) \text {. }
\end{aligned}
$$


Next, it follows from (9) and (27) that

$$
\begin{aligned}
& \operatorname{tr}\left[\eta^{T}\left(t, y(t-\sigma(t)), x\left(t-d_{1}(t)\right)\right)\right. \\
& \left.\times\left(P_{1}+V_{1}\right) \eta\left(t, y(t-\sigma(t)), x\left(t-d_{1}(t)\right)\right)\right] \\
& \leq \lambda_{\max }\left(P_{1}+V_{1}\right)\left[\eta^{T}\left(t, y(t-\sigma(t)), x\left(t-d_{1}(t)\right)\right)\right. \\
& \left.\times \eta\left(t, y(t-\sigma(t)), x\left(t-d_{1}(t)\right)\right)\right] \\
& \leq y^{T}(t-\sigma(t)) \rho_{1} \Sigma_{1}^{T} \Sigma_{1} y(t-\sigma(t)) \\
& +x^{T}\left(t-d_{1}(t)\right) \rho_{1} \Sigma_{2}^{T} \Sigma_{2} x\left(t-d_{1}(t)\right), \\
& \operatorname{tr}\left[\eta^{T}\left(t, y\left(t-d_{2}(t)\right), x(t-\tau(t))\right)\right. \\
& \left.\quad \times\left(P_{2}+V_{2}\right) \eta\left(t, y\left(t-d_{2}(t)\right), x(t-\tau(t))\right)\right] \\
& \leq \lambda_{\max }\left(P_{2}+V_{2}\right)\left[\eta^{T}\left(t, y\left(t-d_{2}(t)\right), x(t-\tau(t))\right)\right. \\
& \left.\quad \times \eta\left(t, y\left(t-d_{2}(t)\right), x(t-\tau(t))\right)\right] \\
& \leq x^{T}(t-\tau(t)) \rho_{2} \Sigma_{4}^{T} \Sigma_{4} x(t-\tau(t)) \\
& +y^{T}\left(t-d_{2}(t)\right) \rho_{2} \Sigma_{3}^{T} \Sigma_{3} y\left(t-d_{2}(t)\right) .
\end{aligned}
$$

Add both sides of (34) and (38) to both sides of (32) and apply (36)-(37); one can obtain that

$$
\mathscr{F} V\left(t, x_{t}, y_{t}\right) \leq \varepsilon^{T}(t) \Upsilon \varepsilon(t)
$$

where $\Upsilon=\phi+T_{1} X T_{1}+T_{2} Y T_{2}+h_{1} N_{1} Z_{1}^{-1} N_{1}^{T}+h_{3} M_{1} Z_{3}^{-1} M_{1}^{T}+$ $h_{5} S_{1} Z_{5}^{-1} S_{1}^{T}+h_{7} E_{1} Z_{7}^{-1} E_{1}^{T}+\Theta$, with $\Theta=\left(d_{1}(t)-h_{1}\right) N_{2} Z_{2}^{-1} N_{2}^{T}+$ $\left(h_{2}-d_{1}(t)\right) N_{3} Z_{2}^{-1} N_{3}^{T}+\left(d_{2}(t)-h_{5}\right) S_{2} Z_{6}^{-1} S_{2}^{T}+\left(h_{6}-\right.$ $\left.d_{2}(t)\right) S_{3} Z_{6}^{-1} S_{3}^{T}+\left(\sigma(t)-h_{3}\right) M_{2} Z_{4}^{-1} M_{2}^{T}+\left(h_{4}-\sigma(t)\right) M_{3} Z_{4}^{-1} M_{3}^{T}+$ $\left(\tau(t)-h_{7}\right) E_{2} Z_{8}^{-1} E_{2}^{T}+\left(h_{8}-\tau(t)\right) E_{3} Z_{8}^{-1} E_{3}^{T}$.

Noting Assumption 2, $\Theta$ can be seen as the convex combination of $N_{2} Z_{2}^{-1} N_{2}^{T}$ and $N_{3} Z_{2}^{-1} N_{3}^{T}$ on $d_{1}(t), M_{2} Z_{4}^{-1} M_{2}^{T}$ and $M_{3} Z_{4}^{-1} M_{3}^{T}$ on $\sigma(t), S_{2} Z_{6}^{-1} S_{2}^{T}$ and $S_{3} Z_{6}^{-1} S_{3}^{T}$ on $d_{2}(t)$, and $E_{2} Z_{8}^{-1} E_{2}^{T}$ and $E_{3} Z_{8}^{-1} E_{3}^{T}$ on $\tau(t)$. Therefore, $\Upsilon<0$ holds if

$$
\begin{gathered}
\Lambda+h_{12} N_{2} Z_{2}^{-1} N_{2}^{T}<0, \\
\Lambda+h_{12} N_{3} Z_{2}^{-1} N_{3}^{T}<0, \\
\Lambda+h_{34} M_{2} Z_{4}^{-1} M_{2}^{T}<0, \\
\Lambda+h_{34} M_{3} Z_{4}^{-1} M_{3}^{T}<0, \\
\Lambda+h_{56} S_{2} Z_{6}^{-1} S_{2}^{T}<0, \\
\Lambda+h_{56} S_{3} Z_{6}^{-1} S_{3}^{T}<0, \\
\Lambda+h_{78} E_{2} Z_{8}^{-1} E_{2}^{T}<0, \\
\Lambda+h_{78} E_{3} Z_{8}^{-1} E_{3}^{T}<0,
\end{gathered}
$$

where

$$
\begin{aligned}
\Lambda=\frac{1}{4}\{\phi & +T_{1} X T_{1}+T_{2} Y T_{2}+h_{1} N_{1} Z_{1}^{-1} N_{1}^{T} \\
& \left.+h_{3} M_{1} Z_{3}^{-1} M_{1}^{T}+h_{5} S_{1} Z_{5}^{-1} S_{1}^{T}+h_{7} E_{1} Z_{7}^{-1} E_{1}^{T}\right\} .
\end{aligned}
$$

By Schur complements, (40) is equivalent to $\phi_{i}<0, i=$ $1,2,3, \ldots, 8$, respectively. Then

$$
\dot{V}\left(t, x_{t}, y_{t}\right)<0 \text {. }
$$

On the other hand, from (27) and Theorem 6 conditions, we note that

$$
\begin{aligned}
\mathbf{V}_{\mathbf{1}}\left(\mathbf{t}_{\mathbf{k}}, \mathbf{x}_{\mathbf{t}}, \mathbf{y}_{\mathbf{t}}\right)= & 2 \sum_{i=1}^{n} \lambda_{i} \int_{0}^{y_{i}\left(t_{k}\right)}\left(f_{i}(s)-\gamma_{i} s\right) d s \\
& +2 \sum_{i=1}^{n} \lambda_{i} \int_{0}^{x_{i}\left(t_{k}\right)}\left(f_{i}(s)-\gamma_{i} s\right) d s \\
= & 2 \sum_{i=1}^{n} \lambda_{i} \int_{0}^{\left\{1-\chi_{i k}\right\} y_{i}\left(t_{k}^{-}\right)}\left(f_{i}(s)-\gamma_{i} s\right) d s \\
& +2 \sum_{i=1}^{n} \lambda_{i} \int_{0}^{\left\{1-\chi_{i k}\right\} x_{i}\left(t_{k}^{-}\right)}\left(f_{i}(s)-\gamma_{i} s\right) d s \\
\leq & 2 \sum_{i=1}^{n} \lambda_{i} \int_{0}^{y_{i}\left(t_{k}^{-}\right)}\left(f_{i}(s)-\gamma_{i} s\right) d s \\
& +2 \sum_{i=1}^{n} \lambda_{i} \int_{0}^{x_{i}\left(t_{k}^{-}\right)}\left(f_{i}(s)-\gamma_{i} s\right) d s \\
= & V_{1}\left(t_{k}^{-}, x_{t}, y_{t}\right) .
\end{aligned}
$$

Moreover, it is obvious that $V_{2}\left(t_{k}, x_{t}, y_{t}\right)=V_{2}\left(t_{k}^{-}, x_{t}, y_{t}\right)$, $V_{3}\left(t_{k}, x_{t}, y_{t}\right)=V_{3}\left(t_{k}^{-}, x_{t}, y_{t}\right)$. Hence, we get $V\left(t_{k}, x_{t}, y_{t}\right) \leq$ $V\left(t_{k}^{-}, x_{t}, y_{t}\right)$. By Lyapunov-Krasovskii stability theorem, the equilibrium point of (8) is stable in the mean square. The proof is completed.

Remark 7. For the UISGRNs (8) without stochastic disturbances, leakage delay, and impulsive effects, it reduces to the model of [27]. And when the model of (8) without impulsive, it reduces to the model of [30]. In addition, it is easy to see that the main theorem obtained above covers the sparse results available in the literature in the concern of only one or two of the complex dynamics generally being involved with GRNs, leakage delays, parameter uncertainties, impulsive effects, and stochastic disturbances.

We give a couple of corollaries below in order to show further that our main result is general enough to cover two cases that have not been investigated in the literature. Hence, they are new and significant. Firstly, for model (6) or the UISGRNs (8) without parameter uncertainties (i.e., $\Delta A(t)=$ $\Delta W(t)=\Delta C(t)=\Delta L(t)=0)$, we have the following corollary. 
Corollary 8. If (7), (9), and Assumption 1 hold, there exist $\mu \geq 0, \lambda \geq 0, \rho_{1}>0, \rho_{2}>0, \chi_{\text {im }} \in[\mathbf{0}, \mathbf{1}], k=0,1, \ldots$, $r+2, i=1, \ldots, n, m \in Z^{+}$, such that the impulsive operator $J_{m}(\cdot)$ satisfies $J_{i m}\left(x_{i}\left(t_{m}\right)\right)=-\chi_{i m} x_{i}\left(t_{m}\right)$. The system (6) is stable in the mean square if there exist real matrices $P_{1}>0, P_{2}>0$, $Q_{i}>0(i=1,2, \ldots, 16), Z_{i}>0(i=1,2, \ldots, 8), V_{1}>0$, and $V_{2}>0$, diagonal matrices $Y_{i}>0(i=1,2, \ldots, 6)$, and any matrices $N_{11}, N_{12}, N_{21}, N_{22}, M_{11}, M_{12}, M_{21}, M_{22}, M_{31}$, $M_{32}, S_{11}, S_{12}, S_{21}, S_{22}, S_{31}, S_{32}, E_{11}, E_{12}, E_{21}, E_{22}, E_{31}$, and $E_{32}$, to satisfy conditions (16)-(25) replaced accordingly by the following:

$$
\begin{aligned}
& \phi_{1,2}=-P_{1} A-T V_{2} A-N_{11}+N_{12}+N_{13}-N_{21}+E_{12}, \\
& \phi_{1,17}=-T V_{2} W+P_{1} W, \quad \phi_{2,15}=-V_{2} A, \\
& \phi_{5,8}=-T V_{1} L+P_{2} L, \quad \phi_{12,16}=-V_{1} L, \\
& \phi_{8,12}=-P_{2} C+T V_{1} C, \\
& \phi_{15,17}=V_{2} W, \\
& T_{1}=\left[\begin{array}{llllllllllllllllllll}
0 & -A & 0 & 0 & 0 & 0 & 0 & 0 & 0 & 0 & 0 & 0 & 0 & 0 & 0 & 0 & W & 0 & 0 & 0
\end{array}\right]^{T}, \\
& T_{2}=\left[\begin{array}{llllllllllllllllllll}
0 & 0 & 0 & L & 0 & 0 & 0 & 0 & 0 & 0 & 0 & -C & 0 & 0 & 0 & 0 & 0 & 0 & 0 & 0
\end{array}\right]^{T} .
\end{aligned}
$$

In the second case, we suppose that there are no stochastic disturbances in the UISGRNs model (8). Hence, model (8) can be reduced to

$$
\begin{aligned}
& \dot{x}(t)=-(A+\Delta A) x\left(t-d_{1}(t)\right) \\
& +(W+\Delta W) f(y(t-\sigma(t))) \\
& \left.\Delta x(t)\right|_{t=t_{k}}=x\left(t_{k}\right)-x\left(t_{k}^{-}\right)=J_{k}\left(x\left(t_{k}^{-}\right)\right) \\
& k \in Z^{+}, \quad t=t_{k}, \\
& \dot{y}(t)=-(C+\Delta C) y\left(t-d_{2}(t)\right)+(L+\Delta L) x(t-\tau(t)) \\
& \left.\Delta y(t)\right|_{t=t_{k}}=y\left(t_{k}\right)-y\left(t_{k}^{-}\right)=J_{k}\left(y\left(t_{k}^{-}\right)\right) \\
& t=t_{k}, \quad k \in Z^{+}, \\
& x_{0}=x(\theta)=\varphi(\theta), \quad y_{0}=y(\theta)=\psi(\theta) \text {, } \\
& \forall \theta \in[-\omega, 0] \text {. }
\end{aligned}
$$

Then we have the following new result.

Corollary 9. If (7) and Assumptions 1 and 2 hold, there exist $\mu \geq 0, \lambda \geq 0, \chi_{\mathrm{im}} \in[\mathbf{0}, \mathbf{1}], k=0,1, \ldots, r+2$, $i=1, \ldots, n, m \in Z^{+}$, such that the impulsive operator $J_{m}(\cdot)$ satisfies $J_{i m}\left(x_{i}\left(t_{m}\right)\right)=-\chi_{i m} x_{i}\left(t_{m}\right)$. The system (45) is stable if there exist real matrices $P_{1}>0, P_{2}>0, Q_{i}>0(i=$ $1,2, \ldots, 16), Z_{i}>0(i=1,2, \ldots, 8), V_{1}>0$, and $V_{2}>0$, diagonal matrices $Y_{i}>0(i=1,2, \ldots, 6)$, and any matrices $N_{11}, N_{12}, N_{21}, N_{22}, M_{11}, M_{12}, M_{21}, M_{22}, M_{31}, M_{32}, S_{11}, S_{12}$,
$S_{21}, S_{22}, S_{31}, S_{32}, E_{11}, E_{12}, E_{21}, E_{22}, E_{31}$, and $E_{32}$, to satisfy conditions (18)-(25) replaced accordingly by the following:

$$
\begin{aligned}
& \phi_{2,2}=-\left(1-d_{1}\right) Q_{1}-2 N_{12}-2 N_{22}+2 M_{32}, \\
& \phi_{5,5}=-(1-\tau) Q_{13}-T Y_{5} \Sigma, \\
& \phi_{9,9}=-(1-\sigma) Q_{5}-T Y_{3} \Sigma, \\
& \phi_{12,12}=-\left(1-d_{2}\right) Q_{9}-T Y_{4} \Sigma .
\end{aligned}
$$

\section{Numerical Examples}

In this section, we present three numerical examples so as to illustrate the usefulness of our results derived in this paper.

Example 1. Let us firstly consider the system (8) with parameters as follows:

$$
\begin{aligned}
& C=\left[\begin{array}{ll}
1 & 0 \\
0 & 1
\end{array}\right], \quad \mathbf{A}=\left[\begin{array}{cc}
1.1 & 0 \\
0 & 1.1
\end{array}\right], \\
& \mathbf{L}=\left[\begin{array}{cc}
0.8 & 0 \\
0 & 0.8
\end{array}\right], \quad \mathbf{W}=\left[\begin{array}{cc}
0.8 & 0 \\
0 & 0.8
\end{array}\right], \\
& \Sigma_{1}=\Sigma_{2}=\left[\begin{array}{cc}
0.1667 & 0 \\
0 & 0.1067
\end{array}\right], \\
& I=\left[\begin{array}{ll}
1 & 0 \\
0 & 1
\end{array}\right], \quad \mathbf{G}_{1}=0.2 * I, \quad \mathbf{G}_{2}=0.2 * I, \\
& \mathbf{H}_{\mathbf{a}}=\mathbf{H}_{\mathbf{w}}=\mathbf{H}_{\mathbf{c}}=\mathbf{H}_{1}=0.02 * I, \\
& F_{1}=0.5 * I, \quad F_{2}=0.6 * I .
\end{aligned}
$$

For convenience, we assume that $\chi_{i m}=1 / 3, d_{1}(t)=0.1+$ $0.05 \sin (t), \sigma(t)=0.2+0.03 \cos (t), d_{2}(t)=0.3+0.01 \cos (t)$, $\tau(t)=0.4+0.05 \cos (t)$, and $\mathbf{f}_{\mathbf{1}}(\mathbf{x})=\mathbf{f}_{\mathbf{2}}(\mathbf{x})=\mathbf{1} /\left(\mathbf{1}+\mathbf{x}^{2}\right)$; then we can obtain

$$
\begin{array}{lll}
h_{1}=0.05, & h_{2}=0.15, & h_{3}=0.17, \\
h_{4}=0.23, & h_{5}=0.29, & h_{6}=0.31, \\
h_{7}=0.35, & h_{8}=0.45, & \\
d_{1}=0.5, & d_{2}=0.6, & \tau=0.7, \\
\sigma=0.8, & \Sigma=\left[\begin{array}{ll}
1 & 0 \\
0 & 1
\end{array}\right], & T=0 .
\end{array}
$$

Using MATLAB LMI control toolbox and by solving the LMIs (16) in Theorem 6, we can obtain the feasible solutions. Due to space limitations, we do not list them here. We find that the delayed UISGRNs (8) are stable in the mean square which is shown in Figure 1.

Example 2. Consider the system (6) with the parameters that are the same as in Example 1 other than

$$
H_{a}=H_{c}=H_{w}=H_{l}=0 .
$$

We can find feasible solutions for the LMIs in Corollary 8 , so the impulsive stochastic GRNs (6) are stable in the mean square which can be shown in Figure 2. 


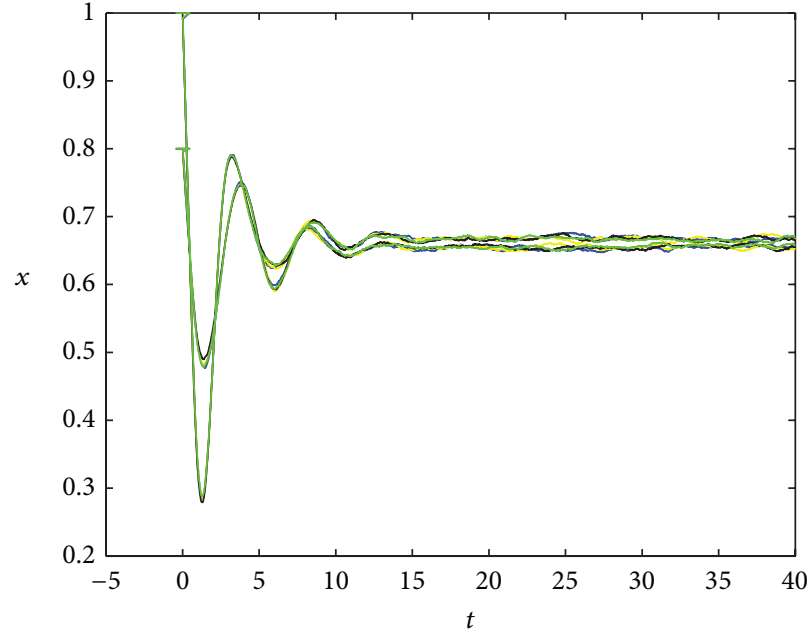

(a)

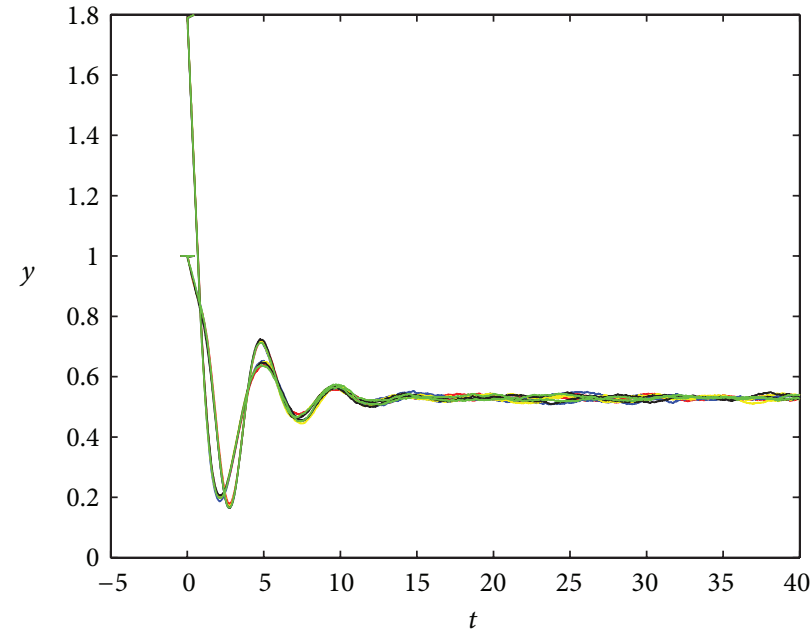

(b)

FIgURE 1: Trajectories of $x(t)$ and $y(t)$ of the genetic network (8) with randomly chosen initial values.

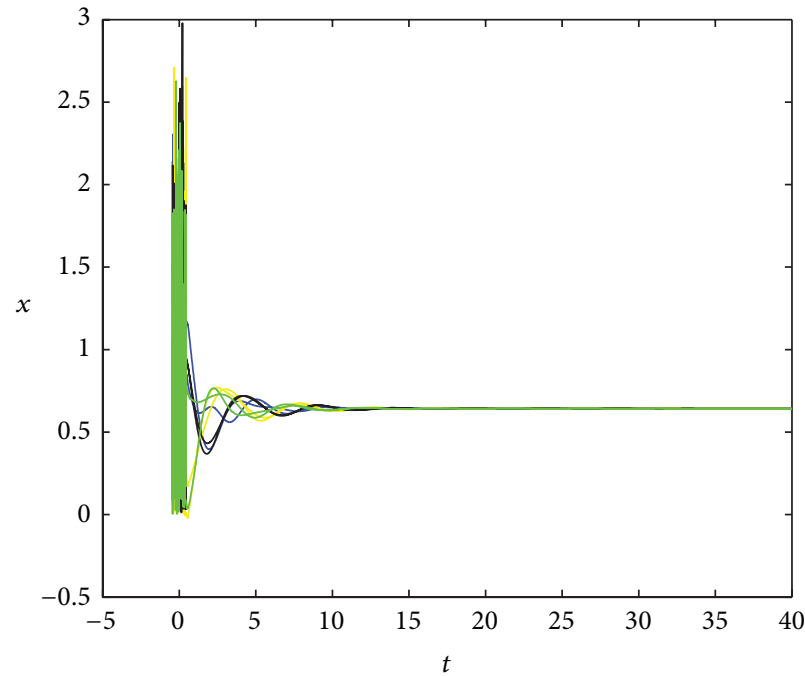

(a)

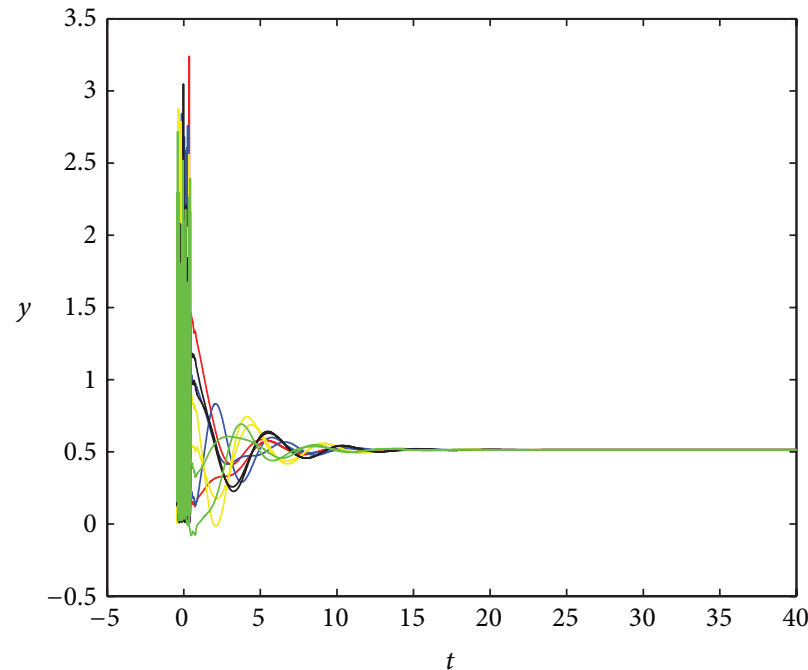

(b)

Figure 2: Trajectories of $x(t)$ and $y(t)$ of the genetic network (6) with randomly chosen initial values.

Example 3. When the parameters in (45) are the same as in Example 1 in addition to

$$
\eta=0 .
$$

It is easy to see that the uncertain impulsive GRNs (45) are stable in the mean square by checking Corollary 9 conditions. Figure 3 shows that the result is valid.

\section{Conclusions}

In this paper, we have investigated the stability problem for a new UISGRNs model with the introduction of leakage delay. By employing the Lyapunov stability theory, free-weighting matrix, and convex combination technique combining with stochastic stability approach and the LMI framework, we have obtained a sufficient condition to justify the stability of the proposed UISGRNs model. The obtained stability condition is expressed in terms of LMIs which can be easily solved by the efficient MATALB LMI toolbox. Finally, numerical examples have been provided to illustrate the usefulness of the derived stability results.

\section{Conflict of Interests}

The authors declare that there is no conflict of interests regarding the publication of this paper.

\section{Acknowledgments}

This work was jointly supported by the Fundamental Research Funds for the Central Universities (JUSRP51317B, 


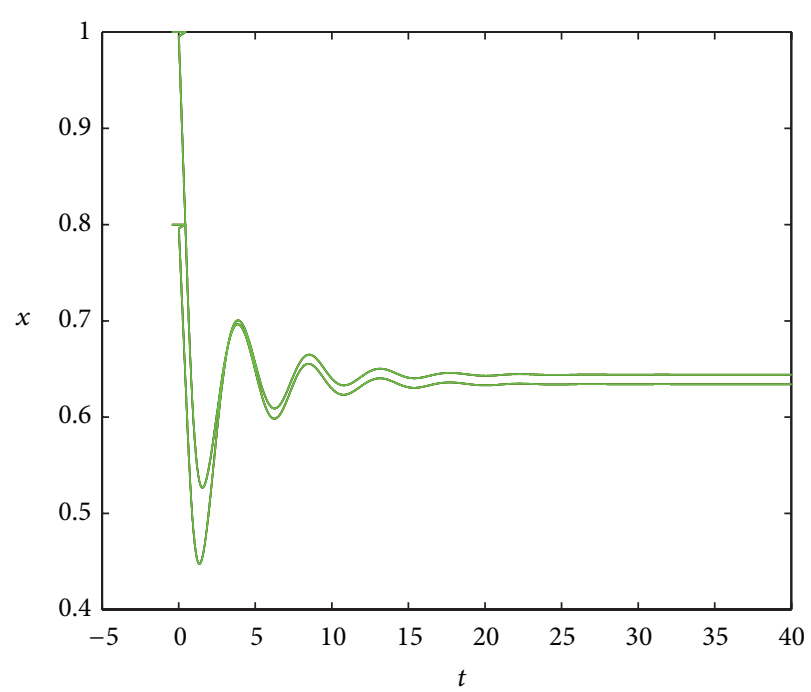

(a)

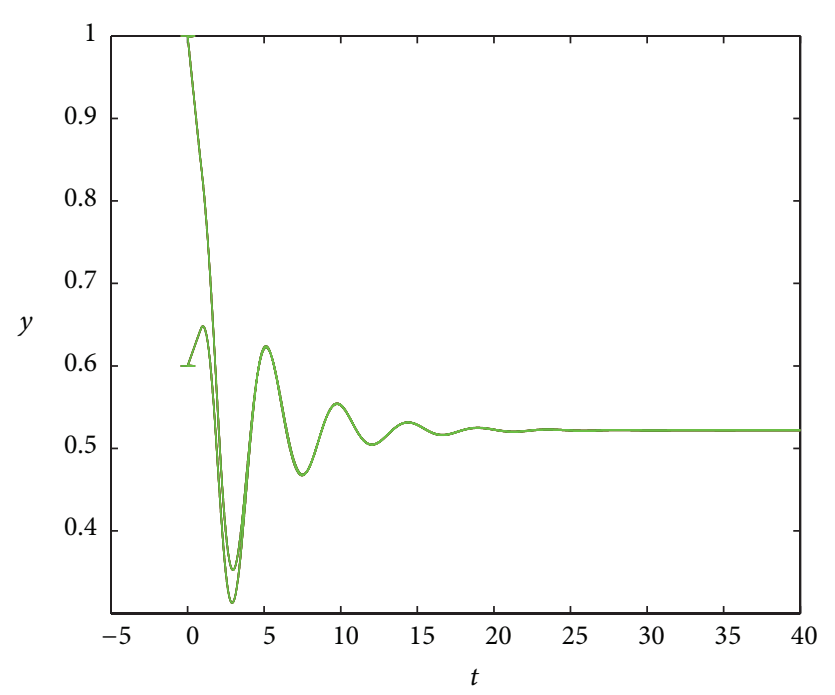

(b)

FIgURE 3: Trajectories of $x(t)$ and $y(t)$ of the genetic network (45) with randomly chosen initial values.

JUSRP211A21), the National Natural Science Foundation of China under Grants 61272530 and 11072059, the Natural Science Foundation of Jiangsu Province of China under Grant BK2012741, and the Specialized Research Fund for the Doctoral Program of Higher Education under Grants 20110092110017 and 20130092110017.

\section{References}

[1] A. Becskel and L. Serrano, "Engineering stability in gene networks by autoregulation," Nature, vol. 405, no. 6786, pp. 590$593,2000$.

[2] M. B. Elowitz and S. Leibier, "A synthetic oscillatory network of transcriptional regulators," Nature, vol. 403, no. 6767, pp. 335$338,2000$.

[3] T. S. Gardner, C. R. Cantor, and J. J. Collins, "Construction of a genetic toggle switch in Escherichia coli," Nature, vol. 403, no. 6767, pp. 339-342, 2000.

[4] H. H. McAdams and L. Shapiro, "Circuit simulation of genetic networks," Science, vol. 269, no. 5224, pp. 650-656, 1995.

[5] L. Chen and K. Aihara, "Stability of genetic regulatory networks with time delay," IEEE Transactions on Circuits and Systems, vol. 49, no. 5, pp. 602-608, 2002.

[6] D. W. Austin, M. S. Allen, J. M. McCollum et al., "Gene network shaping of inherent noise spectra," Nature, vol. 439, no. 7076, pp. 608-611, 2006.

[7] C. Chaouiya, E. Remy, and D. Thieffry, "Petri net modelling of biological regulatory networks," Journal of Discrete Algorithms, vol. 6, no. 2, pp. 165-177, 2008.

[8] S. Hardy and P. N. Robillard, "Modeling and simulation of molecular biology systems using petri nets: modeling goals of various approaches," Journal of Bioinformatics and Computational Biology, vol. 2, no. 4, pp. 595-613, 2004.

[9] W. H. Hsu, "Genetic wrappers for feature selection in decision tree induction and variable ordering in Bayesian network structure learning," Information Sciences, vol. 163, no. 1-3, pp. 103-122, 2004.
[10] N. Friedman, M. Linial, I. Nachman, and D. Pe’er, "Using Bayesian networks to analyze expression data," Journal of Computational Biology, vol. 7, no. 3-4, pp. 601-620, 2000.

[11] A. J. Hartemink, D. K. Gifford, T. S. Jaakkola, and R. A. Young, "Bayesian methods for elucidating genetic regulatory networks," IEEE Intelligent Systems and Their Applications, vol. 17, no. 2, pp. 37-43, 2002.

[12] B. G. Marcot, "Metrics for evaluating performance and uncertainty of Bayesian network models," Ecological Modelling, vol. 230, pp. 50-62, 2012.

[13] R. Somogyi and C. A. Sniegoski, "Modeling the complexity of genetic networks: understanding multigenic and pleiotropic regulation," Complexity, vol. 1, no. 6, pp. 45-63, 1996.

[14] D. Weaver, C. Workman, and G. Stormo, "Modeling regulatory networks with weight matrices," in Proceedings of the Pacific Symposium on Biocomputing (PSB '99), vol. 4, pp. 112-123, Big Island of Hawaii, Hawaii, USA, 1999.

[15] M. Chaves, R. Albert, and E. D. Sontag, "Robustness and fragility of Boolean models for genetic regulatory networks," Journal of Theoretical Biology, vol. 235, no. 3, pp. 431-449, 2005.

[16] H. Bolouri and E. H. Davidson, "Modeling transcriptional regulatory networks," BioEssays, vol. 24, no. 12, pp. 1118-1129, 2002.

[17] H. de Jong, "Modeling and simulation of genetic regulatory systems: a literature review," Journal of Computational Biology, vol. 9, no. 1, pp. 67-103, 2002.

[18] P. Smolen, D. A. Baxter, and J. H. Byrne, "Mathematical modeling of gene networks," Neuron, vol. 26, no. 3, pp. 567-580, 2000.

[19] F. Ren and J. Cao, "Asymptotic and robust stability of genetic regulatory networks with time-varying delays," Neurocomputing, vol. 71, no. 4-6, pp. 834-842, 2008.

[20] W. Zhang, J.-A. Fang, and Y. Tang, "New robust stability analysis for genetic regulatory networks with random discrete delays and distributed delays," Neurocomputing, vol. 74, no. 14-15, pp. 2344-2360, 2011.

[21] M. Hu, J. Cao, and Y. Yang, "Stability of genetic networks with hybrid regulatory mechanism," Arabian Journal of Mathematics, vol. 1, no. 3, pp. 319-328, 2012. 
[22] K. Gopalsamy, "Leakage delays in BAM," Journal of Mathematical Analysis and Applications, vol. 325, no. 2, pp. 1117-1132, 2007.

[23] D. Zhang and L. Yu, "Passivity analysis for stochastic Markovian switching genetic regulatory networks with time-varying delays," Communications in Nonlinear Science and Numerical Simulation, vol. 16, no. 8, pp. 2985-2992, 2011.

[24] T. Tian, K. Burrage, P. M. Burrage, and M. Carletti, "Stochastic delay differential equations for genetic regulatory networks," Journal of Computational and Applied Mathematics, vol. 205, no. 2, pp. 696-707, 2007.

[25] K. A. Pavlov, D. A. Chistiakov, and V. P. Chekhonin, "Genetic determinants of aggression and impulsivity in humans," Journal of Applied Genetics, vol. 53, no. 1, pp. 61-82, 2012.

[26] F. Li and J. Sun, "Asymptotic stability of a genetic network under impulsive control," Physics Letters A, vol. 374, no. 31-32, pp. 31773184, 2010.

[27] H. Wu, X. Liao, W. Feng, S. Guo, and W. Zhang, "Robust stability for uncertain genetic regulatory networks with interval timevarying delays," Information Sciences, vol. 180, no. 18, pp. 35323545, 2010.

[28] W. Wang, S. Zhong, S. Nguang, and F. Liu, "Novel delaydependent stability criterion for uncertain genetic regulatory networks with interval time-varying delays," Neurocomputing, vol. 121, pp. 170-178, 2013.

[29] X. Li, R. Rakkiyappan, and C. Pradeep, "Robust $\mu$-stability analysis of Markovian switching uncertain stochastic genetic regulatory networks with unbounded time-varying delays," Communications in Nonlinear Science and Numerical Simulation, vol. 17, no. 10, pp. 3894-3905, 2012.

[30] G. Wang and J. Cao, "Robust exponential stability analysis for stochastic genetic networks with uncertain parameters," Communications in Nonlinear Science and Numerical Simulation, vol. 14, no. 8, pp. 3369-3378, 2009.

[31] R. Sakthivel, R. Raja, and S. M. Anthoni, "Asymptotic stability of delayed stochastic genetic regulatory networks with impulses," Physica Scripta, vol. 82, no. 5, Article ID 055009, 2010.

[32] C. Li, L. Chen, and K. Aihara, "Stability of genetic networks with SUM regulatory logic: lur'e system and LMI approach," IEEE Transactions on Circuits and Systems, vol. 53, no. 11, pp. 24512458, 2006.

[33] S. Blythe, X. Mao, and X. Liao, "Stability of stochastic delay neural networks," Journal of the Franklin Institute, vol. 338, no. 4, pp. 481-495, 2001. 


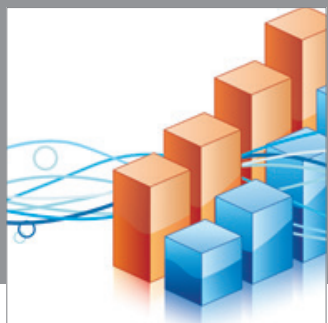

Advances in

Operations Research

mansans

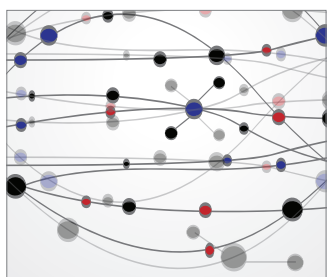

The Scientific World Journal
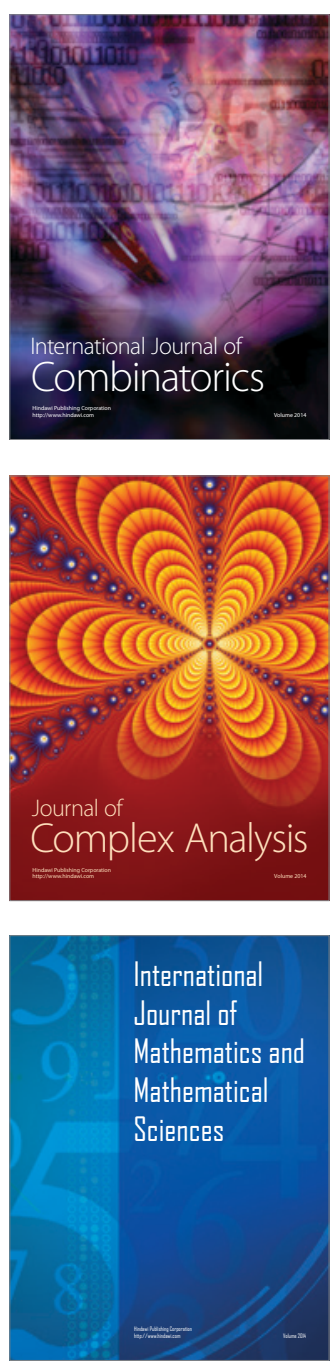


Submit your manuscripts at http://www.hindawi.com
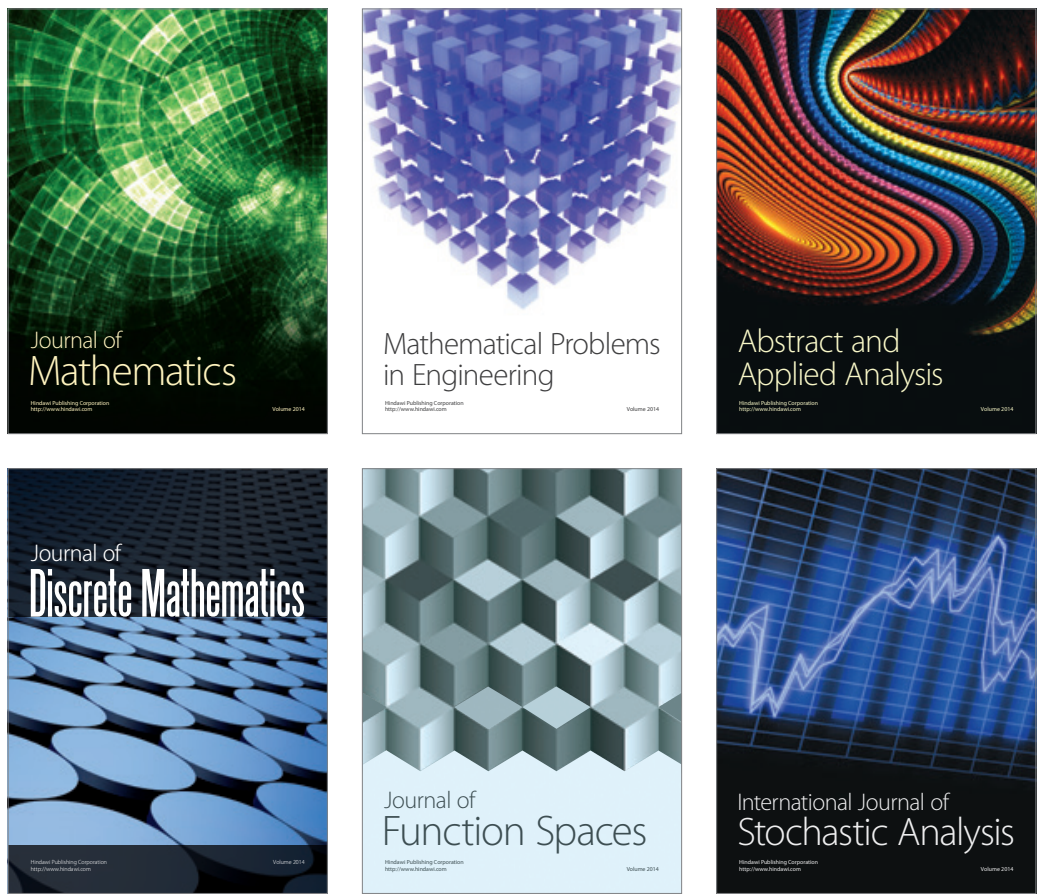

Journal of

Function Spaces

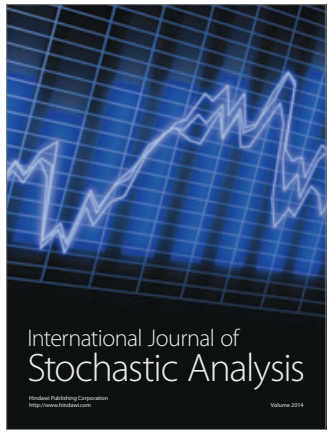

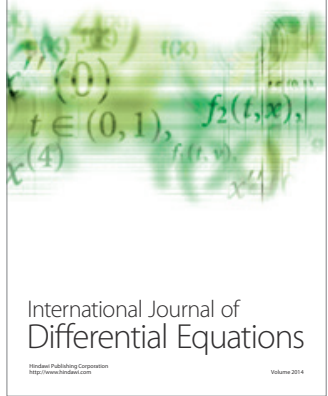
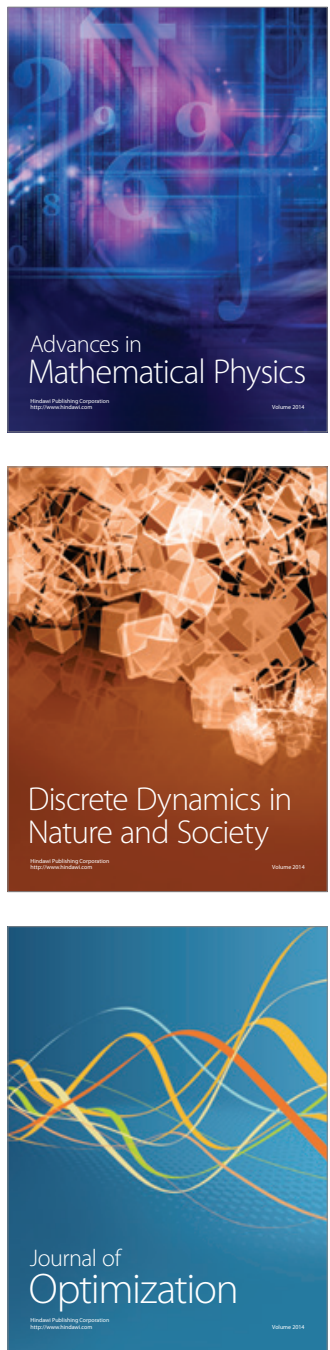